\title{
First stars XI. Chemical composition of the extremely metal-poor dwarfs in the binary CS 22876-032
}

\author{
J. I. González Hernández ${ }^{1,2}$, P. Bonifacio ${ }^{1,2,3}$, H.-G. Ludwig ${ }^{1,2}$, E. Caffau ${ }^{2}$, M. Spite ${ }^{2}$, F. Spite ${ }^{2}$, R. Cayrel ${ }^{2}$, \\ P. Molaro ${ }^{2,3}$, V. Hill ${ }^{2}$, P. François ${ }^{2}$, B. Plez ${ }^{5}$, T. C. Beers ${ }^{4}$, T. Sivarani ${ }^{4}$, J. Andersen ${ }^{6,7}$, B. Barbuy ${ }^{8}$, \\ E. Depagne ${ }^{9}$, B. Nordström ${ }^{6}$, and F. Primas ${ }^{10}$
}

1 CIFIST Marie Curie Excellence Team

2 GEPI, Observatoire de Paris, CNRS, Université Paris Diderot, Place Jules Janssen, 92190 Meudon, France e-mail: [Jonay.Gonzalez-Hernandez;Piercarlo.Bonifacio;Hans.Ludwig;Elisabetta.Caffau;Roger.Cayrel; Monique.Spite;Francois.Spite;Vanessa.Hill;Patrick.Francois]@obspm.fr

3 Istituto Nazionale di Astrofisica - Osservatorio Astronomico di Trieste, via Tiepolo 11, 34143 Trieste, Italy e-mail:molaro@ts.astro.it

4 Department of Physics and Astronomy, CSCE: Center for the Study of Cosmic Evolution, and JINA: Joint Institute for Nuclear Astrophysics, Michigan State University, E. Lansing, MI 48824, USA

e-mail: [thirupati; beers] apa.msu.edu

5 GRAAL, Université de Montpellier II, 34095 Montpellier Cedex 05, France e-mail: Bertrand.Plez@graal.univ-montp2.fr

6 The Niels Bohr Institute, Astronomy, Juliane Maries Vej 30, 2100 Copenhagen, Denmark e-mail: [ja;birgitta]@astro.ku.dk

7 Nordic Optical Telescope, Apartado 474, 38700 Santa Cruz de La Palma, Spain e-mail: ja@not.iac.es

8 Universidade de Sao Paulo, Departamento de Astronomia, Rua do Matao 1226, 05508-900 Sao Paulo, Brazil e-mail: barbuy@astro.iag.usp.br

9 Las Cumbres Observatory, Goleta, CA 93117, USA

e-mail: edepagne@lcogt.net

10 European Southern Observatory (ESO), Karl-Schwarschild-Str. 2, 85749 Garching b. München, Germany e-mail: fprimas@eso.org

Received 12 October 2007 / Accepted 6 December 2007

\section{ABSTRACT}

Context. Unevolved metal-poor stars constitute a fossil record of the early Galaxy, and can provide invaluable information on the properties of the first generations of stars. Binary systems also provide direct information on the stellar masses of their member stars. Aims. The purpose of this investigation is a detailed abundance study of the double-lined spectroscopic binary CS 22876-032, which comprises the two most metal-poor dwarfs known.

Methods. We used high-resolution, high-S/N ratio spectra from the UVES spectrograph at the ESO VLT telescope. Long-term radialvelocity measurements and broad-band photometry allowed us to determine improved orbital elements and stellar parameters for both components. We used OSMARCS 1D models and the TURBOSPECTRUM spectral synthesis code to determine the abundances of Li, O, $\mathrm{Na}, \mathrm{Mg}, \mathrm{Al}, \mathrm{Si}, \mathrm{Ca}, \mathrm{Sc}, \mathrm{Ti}, \mathrm{Cr}, \mathrm{Mn}, \mathrm{Fe}, \mathrm{Co}$ and $\mathrm{Ni}$. We also used the $\mathrm{CO}^{5} \mathrm{BOLD}$ model atmosphere code to compute the 3D abundance corrections, notably for $\mathrm{Li}$ and $\mathrm{O}$.

Results. We find a metallicity of $[\mathrm{Fe} / \mathrm{H}] \sim-3.6$ for both stars, using 1D models with 3D corrections of $\sim-0.1$ dex from averaged 3D models. We determine the oxygen abundance from the near-UV OH bands; the 3D corrections are large, -1 and -1.5 dex for the secondary and primary respectively, and yield $[\mathrm{O} / \mathrm{Fe}] \sim 0.8$, close to the high-quality results obtained from the [OI] $630 \mathrm{~nm}$ line in metal-poor giants. Other $[\alpha / \mathrm{Fe}]$ ratios are consistent with those measured in other dwarfs and giants with similar $[\mathrm{Fe} / \mathrm{H}]$, although Ca and $\mathrm{Si}$ are somewhat low $([\mathrm{X} / \mathrm{Fe}] \lesssim 0)$. Other element ratios follow those of other halo stars. The Li abundance of the primary star is consistent with the Spite plateau, but the secondary shows a lower abundance; 3D corrections are small.

Conclusions. The Li abundance in the primary star supports the extension of the Spite Plateau value at the lowest metallicities, without any decrease. The low abundance in the secondary star could be explained by endogenic Li depletion, due to its cooler temperature. If this is not the case, another, yet unknown mechanism may be causing increased scatter in $A(\mathrm{Li})$ at the lowest metallicities.

Key words. nuclear reactions, nucleosynthesis, abundances - Galaxy: halo - Galaxy: abundances - cosmology: observations stars: Population II

\footnotetext{
^ Based on observations made with the ESO Very Large Telescope at Paranal Observatory, Chile (Large Programme "First Stars", ID 165.N0276(A); P.I. R. Cayrel).

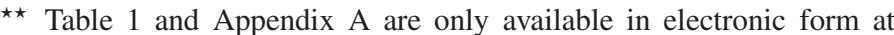
http://www. aanda.org
}

\section{Introduction}

Extremely metal-poor (EMP) stars formed with the chemical composition of the gas in the early Galaxy, and constitute a unique source of information on the first generations of stars. 
Among EMP stars, a special place is held by the dwarfs, which are not subject to the mixing episodes experienced by giants, thus enhancing their value as cosmological probes.

In fact, among these stars the $\mathrm{Li}$ abundance appears to be constant whatever the stellar temperature or metallicity (Spite \& Spite 1982b,a), the Spite plateau. The simplest interpretation of the plateau is that it represents the primordial $\mathrm{Li}$ abundance, i.e., it reflects the amount of Li formed in the first minutes of the existence of the Universe. If so, $\mathrm{Li}$ can be used as a "baryometer", a tool to measure the baryonic density of the Universe, since this is the only cosmological parameter upon which the primordial $\mathrm{Li}$ abundance depends.

The independent determination of the baryonic density from the fluctuations of the cosmic microwave background (CMB) by the WMAP satellite (Spergel et al. 2003, 2007) and other CMB experiments measuring fluctuations on smaller angular scales, such as the VSA (Rebolo et al. 2004; Grainge et al. 2003), ACBAR (Kuo et al. 2004) and CBI (Pearson et al. 2003) experiments, implies a primordial $\mathrm{Li}$ abundance which is at least a factor of 3-4 larger than that observed on the Spite plateau, creating a conflict with the traditional interpretation of the plateau.

In Paper VII in this series (Bonifacio et al. 2007) we investigated the Spite plateau at the lowest metallicities (down to $[\mathrm{Fe} / \mathrm{H}]=-3.3$ ) and found marginal evidence that at these low metallicities there could be an increased scatter or even a sharp drop in the Li abundance. It is therefore of great interest to explore the Li abundance in stars of even lower metallicity.

The star CS 22876-032 was identified in the first paper reporting results of the HK objective-prism survey by Beers et al. (1985), who noted that it had the weakest Ca II K line in the lowmetallicity sample, suggesting that it could be as metal-deficient as the record holder at that time, the giant CD $-38^{\circ} 245$ (Bessell \& Norris 1984). CS 22876-032 had already been observed in the objective-prism survey of Slettebak \& Brundage (1971), who classified it as an A-type peculiar star and noted its weak and diffuse Balmer lines. Having assigned to this star a much earlier spectral type, they did not conclude that the weakness of the $\mathrm{Ca}$ II $\mathrm{K}$ line was indeed due to an extremely low metallicity.

At the conference "Chemical and Dynamical Evolution of Galaxies" in 1989 (Bonifacio et al. 1990), P. Molaro announced that high-resolution spectra from the CASPEC spectrograph at the ESO $3.6 \mathrm{~m}$ telescope indicated $[\mathrm{Fe} / \mathrm{H}] \sim-4.3$ for CS 22876-032. However, just afterwards Nissen (1989) discovered, from simlar resolution spectra, that the star is a doublelined spectroscopic binary. The spectra acquired by Molaro were obtained at a single-lined phase, and the abundance analysis of CS 22876-032 by Molaro \& Castelli (1990) assumed that it was a single star. Thus, veiling was neglected, the adopted temperature was too low, and the measured $[\mathrm{Fe} / \mathrm{H}]$ was therefore a lower limit to the metallicity of the system.

Although CS $22876-032$ is relatively bright $(V=12.84)$ for an EMP star, it took another ten years before a sufficient number of high-resolution spectra had been accumulated to allow determination of the orbital parameters of this system, and to perform a consistent chemical analysis. Norris et al. (2000) found the orbital period to be 424.7 days and the metallicity of the system $[\mathrm{Fe} / \mathrm{H}]=-3.71$. In spite of the upward revision of the metallicity, partly due to the different solar Fe abundance assumed $\left(\log \epsilon(\mathrm{X})_{\odot}=7.50\right.$ instead of 7.63 in Molaro \& Castelli 1990), the two stars in CS 22876-032 remain the most metal-poor dwarfs known.

Thus, the CS 22876-032 system constitutes a unique fossil, recording the chemical composition of the early Galaxy. Moreover, it allows a measurement of the $\mathrm{Li}$ abundance which probes the Spite plateau at a lower metallicity than any other known dwarfs. Note that, despite the extremely low iron abundance $([\mathrm{Fe} / \mathrm{H}]=-5.4)$, the star HE 1327-2326 (Frebel et al. 2005) has very high $\mathrm{C}, \mathrm{N}$ and $\mathrm{O}$ abundances, so its global metallicity, Z, is considerably higher than that of CS 22876-032. It has also been shown recently that this star is most likely a slightly evolved subgiant, not a dwarf.

In this paper we use high-resolution, high- $\mathrm{S} / \mathrm{N}$ ratio spectra from the ESO Kueyen $8.2 \mathrm{~m}$ telescope and the UVES spectrograph to improve the orbital solution and perform a complete chemical analysis of the two stars that comprise CS 22876-032. With respect to the Norris et al. (2000) analysis, our superior S/N ratio and larger spectral coverage permit measurement of abundances for many more elements, and, most importantly, for both components; the Norris et al. (2000) analysis of the secondary star was limited to Fe.

\section{Observations and data reduction}

Spectroscopic observations of the CS 22876-032 were carried out with the UV-Visual Echelle Spectrograph (UVES, Dekker et al. 2000) at the European Southern Observatory (ESO), Observatorio Cerro Paranal, using the $8.2 \mathrm{~m}$ VLT-Kuyen telescope on 2000 July 19, 20, August 3, 11, and October 17, 20, and 2001 November 7, 8 and 9, covering the spectral region from $300.0 \mathrm{~nm}$ to $1040.0 \mathrm{~nm}$. Most of the observations were made with a projected slit width of $1^{\prime \prime}$ at a resolving power $\lambda / \delta \lambda \sim 43000$. The spectra were reduced in a standard manner using the UVES reduction package within the MIDAS environment. The signal-to-noise ratio per pixel varies from 25 at $312.0 \mathrm{~nm}, 50$ at $330.0 \mathrm{~nm}$ up to 150 or higher above $410.0 \mathrm{~nm}$.

\section{Revised orbital parameters}

We derived radial velocities from the UVES spectra by fitting a Gaussian to several unblended spectral lines within the IRAF ${ }^{1}$ context. Table 1 shows the radial velocities and $1-\sigma$ errors estimated from the dispersion of the measurements of different stellar lines. We also list other velocity data given by Norris et al. (2000) and references therein, or which we have measured from previously unpublished CASPEC or EMMI spectra of this system.

Here after, we denote the more massive and luminous primary star as Star A, the secondary as Star B.

Our new radial-velocity measurements of CS 22876-032 extend the time coverage of its orbit considerably and permit improvement of the orbital elements relative to those published by Norris et al. (2000). The computed orbital parameters are given in Table 2; Fig. 1 compares the observed radial velocities of both stars with the curves predicted from these orbital elements.

Note especially the improved mass ratio, $M_{\mathrm{B}} / M_{\mathrm{A}}=0.91 \pm$ 0.02 , which provides stringent constraints on the stellar parameters for the two components of the binary as discussed below. Note also that the orbital eccentricity is the lowest found among halo spectroscopic binaries with periods in the range 100-2000 days (see Latham et al. 2002; Goldberg et al. 2002). While this might be a hint that tidal interaction has been strong in this system, perhaps in the pre-main-sequence phase, the separation of the stars has been so large throughout their

1 IRAF is distributed by National Optical Astronomy Observatory, which is operated by the Association of Universities for Research in Astronomy, Inc., under contract with the National Science Foundation. 
Table 2. Orbital elements of CS 22876-032.

\begin{tabular}{lcc}
\hline \hline Parameter & This paper & Norris et al. (2000) \\
\hline$P$ (days) & $424.81 \pm 0.37$ & $424.71 \pm 0.60$ \\
$T_{0}(\mathrm{HJD}-2400000)$ & $48579.8 \pm 7.1$ & $48576.4 \pm 13.5$ \\
$e$ & $0.143 \pm 0.013$ & $0.12 \pm 0.03$ \\
$w(\mathrm{deg})$ & $148.1 \pm 6.4$ & $144.96 \pm 12.4$ \\
$V_{0}\left(\mathrm{~km} \mathrm{~s}^{-1}\right)$ & $-93.11 \pm 0.13$ & $-93.36 \pm 0.28$ \\
$K_{\mathrm{A}}\left(\mathrm{km} \mathrm{s}^{-1}\right)$ & $15.04 \pm 0.26$ & $15.13 \pm 0.51$ \\
$K_{\mathrm{B}}\left(\mathrm{km} \mathrm{s}^{-1}\right)$ & $16.51 \pm 0.27$ & $17.06 \pm 0.56$ \\
$M_{\mathrm{A}} \sin ^{3} i\left(M_{\odot}\right)$ & $0.701 \pm 0.021$ & $0.76 \pm 0.04$ \\
$M_{\mathrm{B}} \sin ^{3} i\left(M_{\odot}\right)$ & $0.639 \pm 0.019$ & $0.68 \pm 0.04$ \\
$M_{\mathrm{B}} / M_{\mathrm{A}}$ & $0.911 \pm 0.022$ & $0.89 \pm 0.04$ \\
$\sigma\left(\mathrm{km} \mathrm{s}^{-1}\right)$ & 1.01 & 1.60 \\
\hline
\end{tabular}

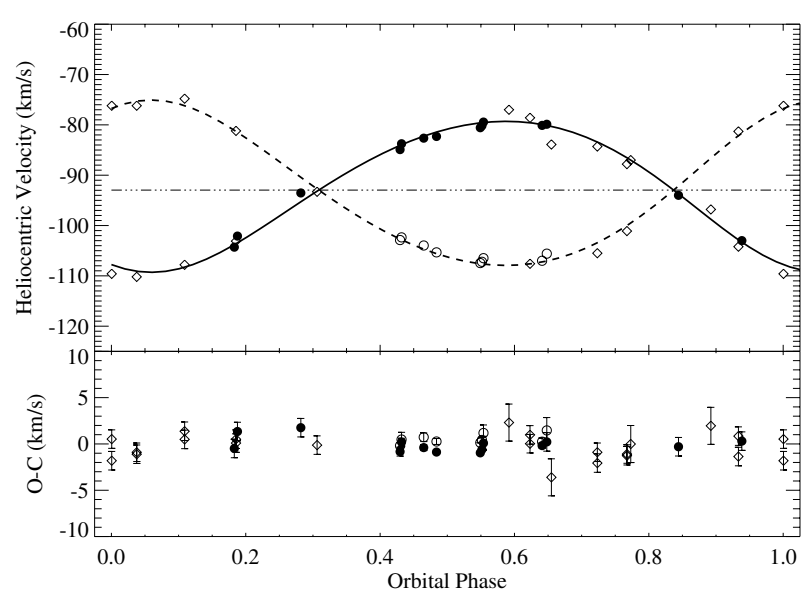

Fig. 1. Upper panel: radial velocities of CS 22876-032 (filled circles: A; open circles: B). Open diamonds: data from Norris et al. (2000). The curves show the orbital solution $(P=425 \mathrm{~d}, e=0.14)$ for Star A (solid) and B (dashed). Dot-dashed horizontal line: centre-of-mass velocity of the system. Lower panel: residuals from the fit.

main-sequence life that this is unlikely to be the cause of the apparent Li depletion we find in star B (see Sect. 6.1).

\section{Model atmospheres}

\subsection{One-dimensional models}

Our analysis used OSMARCS 1D LTE model atmospheres (Gustafsson et al. 1975; Plez et al. 1992; Edvardsson et al. 1993; Asplund et al. 1997; Gustafsson et al. 2003) and the TURBOSPECTRUM spectral synthesis code (Alvarez \& Plez 1998). Models were interpolated in pre-computed grids for a metallicity of $[\mathrm{Fe} / \mathrm{H}]=-3.6$, since this was the final iron abundance (see Sect. 5.2), and with an $\alpha$-element enhancement of $[\alpha / \mathrm{Fe}]=+0.4$ dex. We adopted solar abundances from Grevesse $\&$ Sauval (2000), with the exception of O, for which we adopted $\log \epsilon(\mathrm{O})_{\odot}=8.72$, based on 3D model atmospheres (Ludwig \& Steffen 2007; Caffau et al. 2007b). The code TURBOSPECTRUM is used to determine $1 \mathrm{D}$ element abundances in each component of the binary, either via spectrum synthesis or by comparing the observed equivalent widths of different stellar lines with the theoretical curves of growth (see Sect. 5.2).

\subsection{Three-dimensional models}

In this work we also consider two 3D model atmospheres, which have been computed with the $\mathrm{CO}^{5} \mathrm{BOLD}$ code (Freytag et al. 2002; Wedemeyer et al. 2003), one for each star. The atmospheric parameters are close to those observed for the two stars: $T_{\text {eff }} / \log g /[\mathrm{Fe} / \mathrm{H}]:$ 6550/4.50/-3.0 (A) and 5920/4.50/-3.0 (B). Each model consists of a representative set of snapshots sampling the temporal evolution of the photospheric flow at equal intervals in time. The total time intervals were $2400 \mathrm{~s}$ for the warmer star A, and $9500 \mathrm{~s}$ for the cooler star B. These time intervals should be compared to the convective turn-over time scales. From the hydrodynamical point of view, typical time scales in the models for both components are not much different from that in a solar model, where the convective turn-over time scale amounts to about $500 \mathrm{~s}$. Thus, we sample about five turn-over time scales for the hotter and almost 20 for the cooler component.

The comparison of 3D vs. 1D models depends on which particular 1D model is chosen. We compared each of our 3D models (hereafter denoted as $\langle 3 \mathrm{D}\rangle$, obtained from the mean temperature and pressure structure of the full 3D model), to a corresponding standard hydrostatic 1D model atmosphere (hereafter denoted as $\left.1 \mathrm{D}_{\mathrm{LHD}}\right)$. The $\langle 3 \mathrm{D}\rangle$ model is a temporal and horizontal average of the 3D structure over surfaces of equal (Rosseland) optical depth. It is only dependent on the particular way the 3D model is averaged.

The $1 D_{\text {LHD }}$ model is calculated with a $1 \mathrm{D}$ atmosphere code called LHD. It assumes plane-parallel geometry and employs the same micro-physics (equation-of-state, opacities) as CO ${ }^{5} \mathrm{BOLD}$. Convection is described by mixing-length theory. Somewhat arbitrary choices to be made relate to the value of the mixinglength parameter, which formulation of mixing-length theory to use, and how turbulent pressure is treated in the momentum equation; see Caffau et al. (2007a) for further details. As usual, in the spectral synthesis of the 1D models, a value of the microturbulence has to be adopted. For 1D as well as 3D models the spectral synthesis calculations were performed with the spectrum synthesis code Linfor3 $\mathrm{D}^{2}$.

\section{3. $3 D$ corrections}

There are two main effects that distinguish 3D from 1D models, the average temperature profile and the horizontal temperature fluctuations. We quantify the contribution of both effects by introducing the $3 \mathrm{D}$ correction as: $3 \mathrm{D}-1 \mathrm{D}_{\mathrm{LHD}}$.

The average temperature profile provided by a hydrodynamical simulation is different from that of a $1 \mathrm{D}$ atmosphere assuming radiative equilibrium. This effect is shown in Fig. 2, where the $3 \mathrm{D}$ average temperature profile, plotted as a function of the pressure, is compared to the profiles from $1 \mathrm{D}_{\mathrm{LHD}}$ and MARCS models. As is evident from the plot, the $\langle 3 \mathrm{D}\rangle$ temperature profile is cooler than both $1 \mathrm{D}$ models in the outer photospheric layers for both of the stars. This often-encountered effect in metal-poor atmospheres (Asplund et al. 1999) is particularly important for the oxygen abundances derived from $\mathrm{OH}$ molecules, as the difference is largest precisely in the layers where these lines are formed. The result is that the oxygen abundances become lower in the 3D formulation than in the 1D; we quantify this effect through the 3D correction as defined above.

\footnotetext{
${ }^{2}$ More information on Linfor3D can be found in the following link: http://www.aip.de/ mst/Linfor3D/linfor_3D_manual.pdf
} 


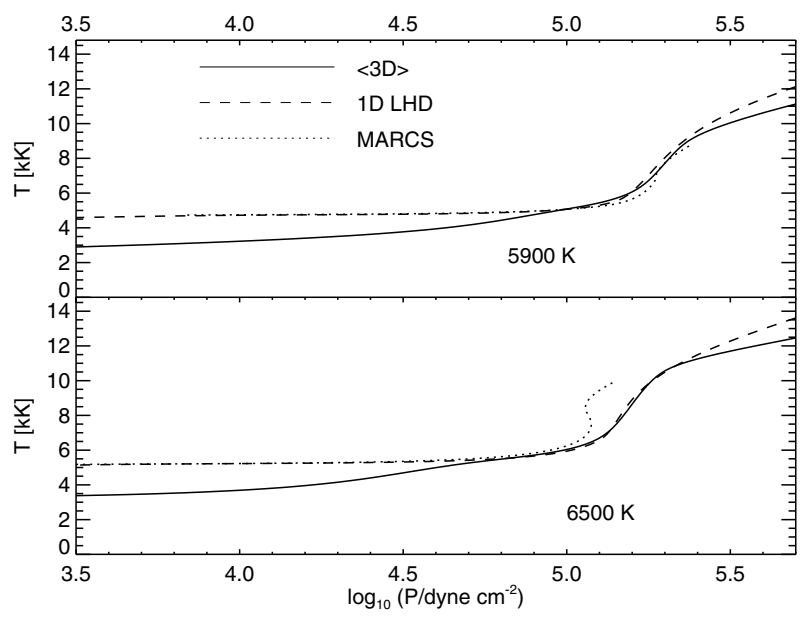

Fig. 2. Average temperature profile of $3 \mathrm{D} \mathrm{CO}^{5} \mathrm{BOLD}$ atmospheric models (solid lines) compared to $1 \mathrm{D}_{\mathrm{LHD}}$ (dashed) and 1D MARCS models (dotted) in stars A (bottom panel) and B (top).

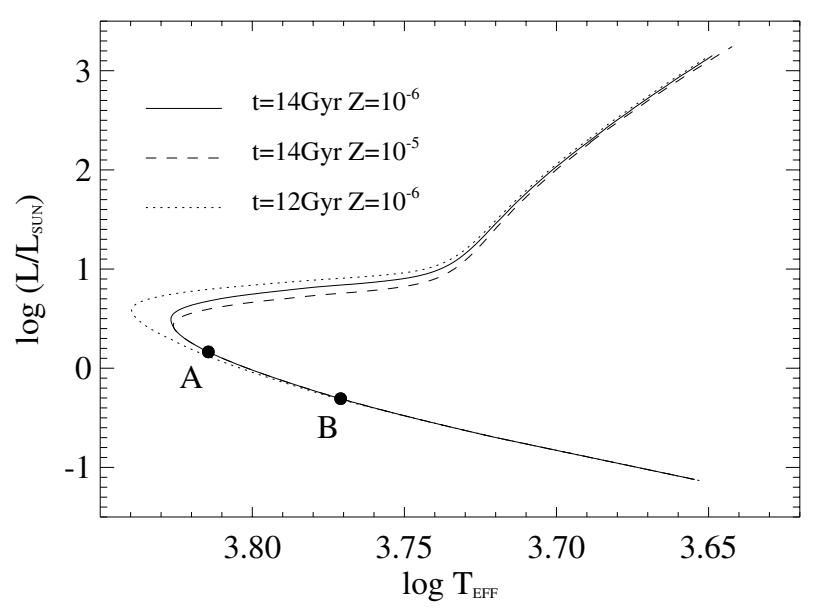

Fig. 3. The two components of CS $22876-032$ on a 14-Gyr isochrone by Chieffi \& Limongi (priv. comm.) for $Z=10^{-6}$ (solid line). For comparison, isochrones for $14 \mathrm{Gyr}$ and $Z=10^{-5}$ (dashed) and $12 \mathrm{Gyr}$ and $Z=10^{-6}$ (dotted) are also shown.

\section{Chemical analysis}

\subsection{Stellar parameters}

The atmospheric parameters of each star in the CS 22876-032 system were estimated from the photometric data available in Norris et al. (1993) and Preston et al. (1991), from whom we adopt $V=12.84, B-V=0.397, U-B=-0.255$ with uncertainties of $0.01,0.02$ and 0.01 respectively. We adopt $E(B-$ $V)=0.00 \pm 0.01$ from Norris et al. (2000) and Schuster et al. $(1993,1996)$. We also extracted, from the $2 \mathrm{MASS}^{3}$ database, $K=11.503 \pm 0.035$ and $J=11.800 \pm 0.041$. The equations derived by Carpenter (2001) to transform from 2MASS magnitudes to the homogenised photometric system of Bessell \& Brett (1988) were then applied.

From the above information it is possible to estimate the reddening-corrected colours $(U-B)_{0},(B-V)_{0},(V-K)_{0}$ and $(J-K)_{0}$, which we use to derive the parameters of both

\footnotetext{
3 The Two Micron All Sky Survey is a joint project of the University of Massachusetts and the Infrared Processing and Analysis Center/California Institute of Technology, funded by the National Aeronautics and Space Administration and the National Science Foundation.
}

components of the binary by comparing with theoretical isochrones. We have chosen the isochrone of Chieffi \& Limongi (priv. commun.) for $14 \mathrm{Gyr}$ and metallicity $Z=10^{-6}$, from which one can compute composite colours from pairs of two models that lie on that isochrone. Thus, the stellar parameters can be derived from the best fit to the observed colours which also satisfy the mass ratio determined from the orbital solution.

We note that the isochrones of Chieffi \& Limongi use the colour transformations based on ATLAS model atmospheres, for this temperature range, and the synthetic colours of Bessell et al. (1998).

The result is shown in Fig. 3, which corresponds to a primary star with $T_{\text {eff, }}=6500 \mathrm{~K}$ and $\log g_{\mathrm{A}}=4.4$ and a secondary star with $T_{\text {eff }, \mathrm{B}}=5900 \mathrm{~K}$ and $\log g_{\mathrm{B}}=4.6$. We checked that a change of $\Delta$ Age $=-2$ Gyr translates into a change of $+25 \mathrm{~K}$ and -0.01 dex for the $T_{\text {eff,A,B }}$ and $\log g_{\mathrm{A}, \mathrm{B}}$ respectively, whereas a variation of the metallicity of $\Delta \log Z=+1$ dex dsoes not have any impact on the resulting stellar parameters (see Fig. 3).

The uncertainties of the stellar parameters were estimated using Monte Carlo techniques. We injected noise in the seven observed quantities, $V, B-V, U-B, K, J, E(B-V)$, and $M_{\mathrm{B}} / M_{\mathrm{A}}$ following Gaussian distributions with standard deviations equal to the errors of these quantities. From these distributions we computed a set of five variables, $(U-B)_{0},(B-V)_{0},(V-K)_{0},(J-K)_{0}$, and $M_{\mathrm{B}} / M_{\mathrm{A}}$ for the 10000 samples. We then found the best fit to each of these set of variables via a $\chi^{2}$ minimisation, defining $\chi^{2}=\sum_{i=1}^{5}\left(f_{i, \text { obs }}-f_{i, \text { mod }}\right)^{2}, f_{i, \text { obs }}$ being the "observed" value for each Monte Carlo simulation and $f_{i \text {,mod }}$ the value extracted from two pairs of points in the theoretical isochrone.

The results of these simulations for the effective temperature and surface gravity of both components are shown in Figs. 4 and 5 respectively. The lowest contour encloses roughly $95.4 \%$ of the 10000 Monte Carlo events, analogous to $2 \sigma$ for a normal distribution. From these simulations we adopted an error, at the $2 \sigma$ level, of $\Delta T_{\text {eff }, \mathrm{A}}=100 \mathrm{~K}$ and $\Delta T_{\text {eff }, \mathrm{B}}=150 \mathrm{~K}$ for the effective temperature, and $\Delta \log g_{\mathrm{A}, \mathrm{B}}=0.1 \mathrm{dex}$ for the surface gravity.

For single stars, the wings of $\mathrm{H} \alpha$ is also a very good temperature indicator (Cayrel 1988; Fuhrmann et al. 1993; van't Veer-Menneret \& Mégessier 1996; Barklem et al. 2002). Adopting the broadening theory of Barklem et al. (2000), we computed $\mathrm{H} \alpha$ profiles for several effective temperatures, using TURBOSPECTRUM. Unfortunately, all our UVES spectra were obtained near maximum line separation, and the velocity difference is of the order of $\sim 30 \mathrm{~km} \mathrm{~s}^{-1}$, which precludes separation of the individual $\mathrm{H} \alpha$ profiles. Therefore, we had to compute a composite spectrum in order to match the observed profile.

Figure 6 compares this synthetic $\mathrm{H} \alpha$ profile with the observed profile for several combinations of effective temperatures $T_{\text {eff,A }}+T_{\text {eff,B }}$. We did not vary $T_{\text {eff,B }}$ because the $\mathrm{H} \alpha$ absorption line of the cooler star B is weaker and severely veiled by the flux of star A, so the combined profile is not sensitive to changes in $T_{\text {eff,B }}$. This comparison seems to confirm our estimate of the effective temperature from the colours, suggesting also that it is on the same scale as the $\mathrm{H} \alpha$-based temperatures.

\subsection{Stellar elemental abundances}

Most of the elemental abundances were determined from equivalent width measurements of selected unblended lines. These were made with an automatic line-fitting procedure based on the algorithms of Charbonneau (1995), which performs both line detection and Gaussian fits to unblended lines. The implementation 

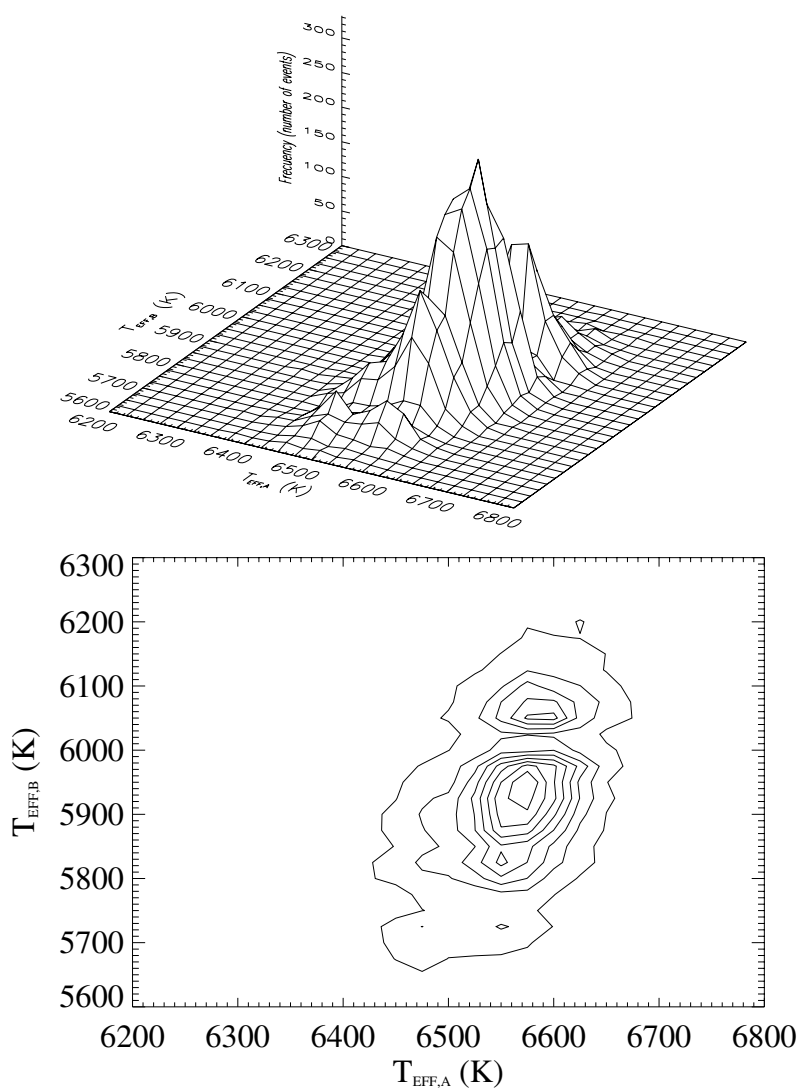

Fig. 4. Distribution of effective temperatures for CS 22876-032 A and $\mathrm{B}$ obtained by Monte Carlo simulations, comparing the observed colours with those from the isochrone in Fig. 3 for the observed mass ratio. The lowest contour encloses $95.4 \%$ of the 10000 simulations.

is the same as described in François et al. (2003). The equivalent widths were then corrected for the appropriate veiling factors and provided as input to TURBOSPECTRUM to determine the abundances. The detailed line-by-line abundances, together with observed $E W \mathrm{~s}$, veiling factors, and atomic data for both components can be found in Table A.1.

The mean abundances for each element, listed in Table 3, are computed using the adopted stellar parameters derived in Sect. 5.1 and a microturbulence of $\xi_{\mathrm{t}}=1.5 \mathrm{~km} \mathrm{~s}^{-1}$. We performed full 3D computations with Linfor3D for the Li doublet and the $\mathrm{OH}$ lines. A full 3D analysis for the hundreds of lines involved in this work is a considerable computational task, well beyond the scope of the present paper. However, we used the $\langle 3 \mathrm{D}\rangle$ models as input to TURBOSPECTRUM to estimate the expected corrections due to the different average temperature profiles of the 3D models. From the full 3D spectrum synthesis performed for $\mathrm{Li}$ and $\mathrm{OH}$, we expect this to be close to the true 3D correction for star $\mathrm{B}$, while we expect significant contributions from the temperature fluctuations in star A. In the following we refer to $\left(\langle 3 \mathrm{D}\rangle-1 \mathrm{D}_{\mathrm{MARCS}}\right)$ as representing the difference between the abundance found by TURBOSPECTRUM using a $\langle 3 \mathrm{D}\rangle$ model and that found using a MARCS 1D model. This is to distinguish them from the true $3 \mathrm{D}$ corrections.

\subsubsection{Veiling corrections}

In a double-lined spectrum the strength of each spectral line, in particular those of the fainter component, is reduced by veiling from the continuum flux of the other star. Thus, the measured equivalent widths for each spectral component must be corrected
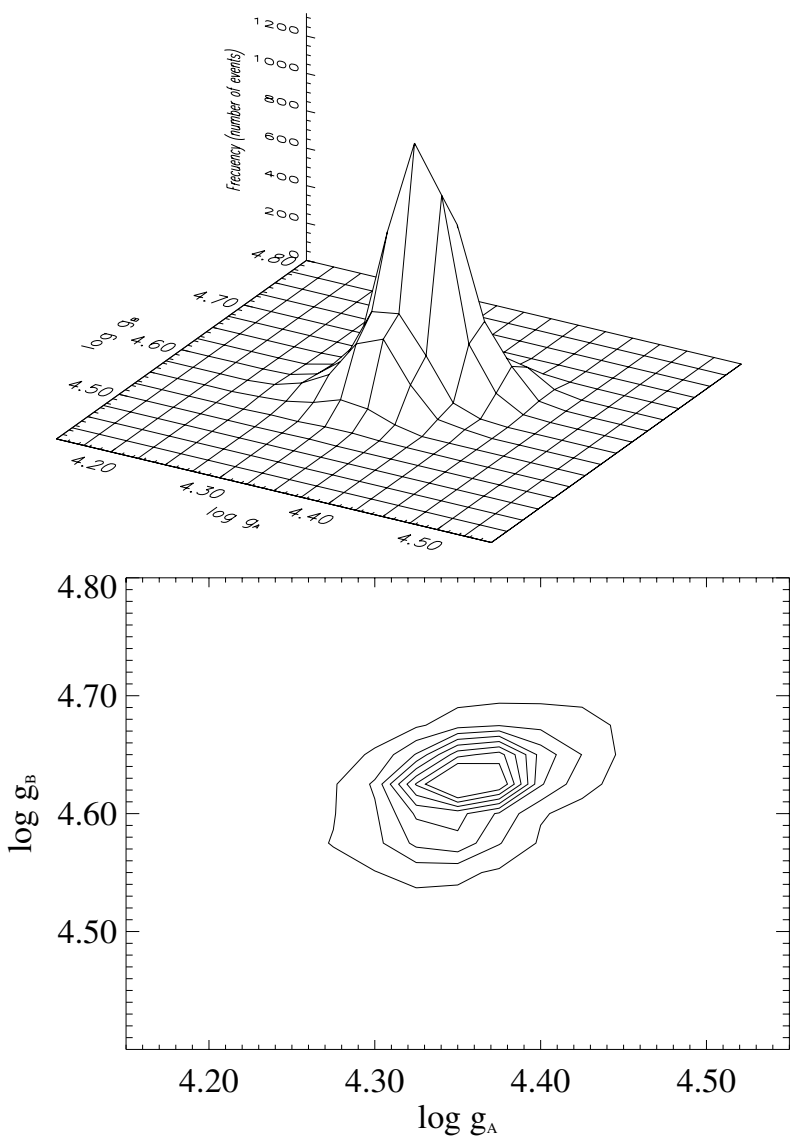

Fig. 5. Same as Fig. 4, for the surface gravities.

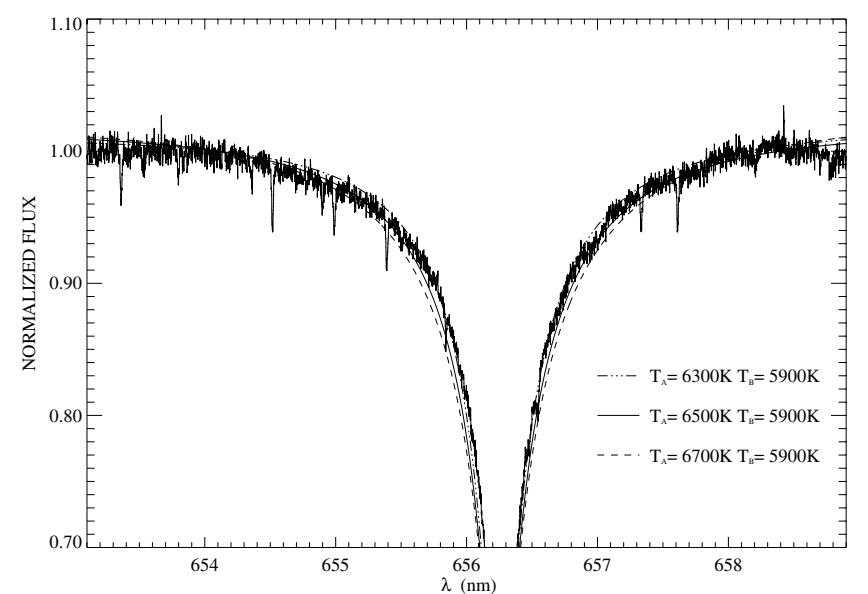

Fig. 6. Computed $\mathrm{H}_{\alpha}$ profiles for three sets of effective temperatures, $6300+5900,6500+5900$ and $6700+5900$, and normalised to the level of the observed UVES spectrum at $645.0 \mathrm{~nm}$.

for this veiling effect, in order to obtain the intrinsic values. The corrected equivalent widths can be estimated by multiplying the observed $E W \mathrm{~s}$ by veiling factors, $f_{\lambda, i}$, which solve the equation $1 / f_{\lambda, \mathrm{A}}+1 / f_{\lambda, \mathrm{B}}=1.0$ and where $f_{\lambda, \mathrm{B}} / f_{\lambda, \mathrm{A}}$ is the primary-tosecondary luminosity ratio. The values $f_{\lambda, i}$ are wavelength dependent and can be estimated theoretically by computing the flux of each stellar component, taking into account the ratio of the stellar radii. Thus, the luminosity ratio can be expressed as $f_{\lambda, \mathrm{B}} / f_{\lambda, \mathrm{A}}=F_{\lambda, \mathrm{A}} / F_{\lambda, \mathrm{B}} \times\left(R_{\mathrm{A}} / R_{\mathrm{B}}\right)^{2}$, where $F_{\lambda, i}$ and $R_{i}$ are the flux and radius of each star. 
Table 3. Element abundances of CS 22876-032. The Solar O abundance is adopted from the 3D measurement of atomic lines of Ludwig \& Steffen (2007); the other Solar abundances from Grevesse \& Sauval (2000). $[\mathrm{X} / \mathrm{H}]$ and $[\mathrm{X} / \mathrm{Fe}]$ are LTE values; $[\mathrm{X} / \mathrm{Fe}]$ refers to $\mathrm{Fe} \mathrm{I}$ for OH and neutral species, to Fe II for ionised species. The 3D abundance corrections $\triangle_{3 \mathrm{D}-1 \mathrm{D}}$ were determined with TURBOSPECTRUM from the $\langle 3 \mathrm{D}\rangle$ and $1 \mathrm{D}$ models, except for $\mathrm{O}$ and $\mathrm{Li}$, where a full 3D analysis was performed and we give two values: the abundance correction 3D - $\langle 3 \mathrm{D}\rangle$ (" $\langle 3 \mathrm{D}\rangle$ ") and "1D" = $3 \mathrm{D}-1 \mathrm{D}_{\mathrm{LHD}}$ (see text). $\sigma$ is the standard deviation of the results from the $n$ lines (next column; if $n=1$, the wavelength of the line in nm is given). See text for details on the measurement of $\mathrm{Li}, \mathrm{O}, \mathrm{Sc}$, and Co.

\begin{tabular}{|c|c|c|c|c|c|c|c|c|c|c|c|}
\hline Species & $\log \epsilon(\mathrm{X})_{\odot}$ & {$[\mathrm{X} / \mathrm{H}]_{\mathrm{A}}$} & {$[\mathrm{X} / \mathrm{Fe}]_{\mathrm{A}}$} & $\Delta_{3 \mathrm{D}-1 \mathrm{D}, \mathrm{A}}$ & $\sigma_{\mathrm{A}}$ & $n_{\mathrm{A}}$ & {$[\mathrm{X} / \mathrm{H}]_{\mathrm{B}}$} & {$[\mathrm{X} / \mathrm{Fe}]_{\mathrm{B}}$} & $\Delta_{3 \mathrm{D}-1 \mathrm{D}, \mathrm{B}}$ & $\sigma_{\mathrm{B}}$ & $\overline{n_{\mathrm{B}}}$ \\
\hline Li I & - & 2.22 & - & $\begin{array}{l}\langle 3 \mathrm{D}\rangle:-0.17 \\
1 \mathrm{D}:-0.19\end{array}$ & 0.01 & 670.8 & 1.75 & - & $\begin{array}{l}\langle 3 D\rangle:-0.02 \\
1 D:-0.29\end{array}$ & 0.04 & 670.8 \\
\hline $\mathrm{O}(\mathrm{OH})$ & 8.72 & -1.52 & 2.14 & $\begin{array}{l}\langle 3 \mathrm{D}\rangle:-0.64 \\
1 \mathrm{D}:-1.49\end{array}$ & 0.04 & 4 & -1.75 & 1.81 & $\begin{array}{l}\langle 3 \mathrm{D}\rangle: 0.00 \\
1 \mathrm{D}:-0.92\end{array}$ & 0.09 & 9 \\
\hline $\mathrm{NaI}$ & 6.33 & -3.69 & -0.03 & -0.01 & 0.03 & 2 & -3.79 & -0.22 & 0.02 & 0.05 & 589.5 \\
\hline $\mathrm{Mg} \mathrm{I}$ & 7.58 & -3.11 & 0.55 & 0 & 0.18 & 9 & -3.14 & 0.43 & 0.09 & 0.24 & 9 \\
\hline $\mathrm{Al} \mathrm{I}$ & 6.47 & -3.89 & -0.23 & -0.02 & 0.22 & 2 & -3.75 & -0.18 & 0.04 & 0.22 & 2 \\
\hline Si I & 7.55 & -3.75 & -0.09 & -0.01 & 0.02 & 390.5 & -3.48 & 0.09 & 0.07 & 0.04 & 390.5 \\
\hline $\mathrm{Ca} \mathrm{I}$ & 6.36 & -3.65 & 0.01 & -0.07 & 0.02 & 422.6 & -3.68 & -0.11 & -0.04 & 0.04 & 422.6 \\
\hline Ca II & 6.36 & -3.68 & -0.18 & 0.10 & 0.14 & 3 & -3.81 & -0.54 & 0.23 & 0.11 & 2 \\
\hline Sc II & 3.17 & -3.73 & -0.22 & 0 & 0.18 & 2 & -3.57 & -0.30 & 0.04 & 0.11 & 2 \\
\hline Ti II & 5.02 & -3.25 & 0.25 & -0.01 & 0.34 & 19 & -3.45 & -0.18 & 0.09 & 0.23 & 12 \\
\hline Cr I & 5.67 & -3.79 & -0.13 & -0.07 & 0.12 & 5 & -3.92 & -0.35 & -0.08 & 0.35 & 4 \\
\hline Cr II & 5.67 & -3.38 & 0.12 & 0.08 & 0.10 & 313.2 & -3.30 & -0.03 & 0.24 & 0.30 & 313.2 \\
\hline Mn II & 5.39 & -4.01 & -0.51 & 0.09 & 0.17 & 344.1 & -3.86 & -0.59 & 0.14 & 0.37 & 344.1 \\
\hline $\mathrm{Fe} \mathrm{I}$ & 7.50 & -3.66 & 0 & -0.12 & 0.12 & 38 & -3.57 & 0 & -0.07 & 0.21 & 37 \\
\hline Fe II & 7.50 & -3.51 & 0 & 0.09 & 0.25 & 13 & -3.27 & 0 & 0.18 & 0.31 & 9 \\
\hline Co I & 4.92 & -2.91 & 0.75 & -0.13 & 0.07 & 7 & -2.92 & 0.65 & -0.07 & 0.08 & 4 \\
\hline Ni I & 6.25 & -3.49 & 0.17 & -0.19 & 0.18 & 12 & -3.45 & 0.12 & -0.08 & 0.16 & 12 \\
\hline
\end{tabular}

For consistency with the isochrone colours, we use version 9 of the ATLAS code (Kurucz 1993a, 2005a) in its Linux version (Sbordone et al. 2004; Sbordone 2005) to compute model atmospheres and fluxes for each star by adopting the $T_{\text {eff }}$ and $\log g$ derived in Sect. 5.1. We used the "NEW" Opacity Distribution Functions (Castelli \& Kurucz 2003), with $1 \mathrm{~km} \mathrm{~s}^{-1}$ micro-turbulence, a mixing-length parameter $\alpha_{\mathrm{MLT}}$ of 1.25 , and no overshooting. The formulation of the mixing length is different between MARCS and ATLAS; that used in our MARCS models roughly corresponds to $\alpha_{\mathrm{MLT}} \sim 1.1$ in the ATLAS formulation.

The veiling factors thus computed differ from the MARCS+TURBOSPECTRUM factors on average by 0.02 which, for a line on the linear part of the curve of growth, translates into a difference in abundance of 0.009 dex, which is negligible within the accuracy of our analysis. This allowed us to compute quickly veiling factors for different combinations of the parameters of the two stars, which were then used to estimate the associated uncertainties. For consistency, we used the ATLAS veiling factors throughout.

The ratio of the stellar radii was extracted from the theoretical isochrone, being $R_{\mathrm{A}} / R_{\mathrm{B}}=1.4$. The derived veiling factors lie in the range $f_{\lambda, \mathrm{A}}=1.29-1.37\left(f_{\lambda, \mathrm{B}}=4.47-3.70\right)$ in the spectral region $\lambda \lambda 300.0-700.0 \mathrm{~nm}$. These estimates also compare well with those used by Norris et al. (2000), although they adopted fixed values for large spectral regions. In particular, they used $f_{\lambda, \mathrm{A}}=1.28$ and $f_{\lambda, \mathrm{B}}=4.60$ for all Fe lines between 370 and $440 \mathrm{~nm}$, whereas we used $f_{\lambda, \mathrm{A}}=1.28-1.31$ and $f_{\lambda, \mathrm{B}}=4.19-4.58$ in that spectral region (see Table A.1).

In addition to the $1 \mathrm{D}$ veiling factors computed for the effective temperatures of both stars, we also calculated the $\langle 3 \mathrm{D}\rangle$ veiling factors using the $\langle 3 \mathrm{D}\rangle$ atmospheric models (whose temperature structure is different from that of the OSMARCS 1D models). These veiling factors were adopted to properly correct the EWs given as input to the TURBOSPECTRUM code to estimate the $\left(\langle 3 \mathrm{D}\rangle-1 \mathrm{D}_{\text {MARCS }}\right)$ abundance corrections (see Sect. 5.2). In addition, we note that the $3 \mathrm{D}-1 \mathrm{D}_{\mathrm{LHD}}$ correction (see Sect. 4.2), computed only for Li and OH lines, does not consider different veiling factors for 1D and 3D models. In this case, we computed only the $3 \mathrm{D}$ veiling factors using the continuum flux provided by the full 3D model of each star in the spectral region close to these lines. Thus, these $3 \mathrm{D}$ veiling factors were applied to the observed $E W \mathrm{~s}$, and the resulting $E W \mathrm{~s}$ were used to compute the $3 \mathrm{D}-1 \mathrm{D}_{\mathrm{LHD}}$ corrections reported in Table 3.

\subsubsection{Uncertainties in the abundance analysis}

The abundance measurements are dependent on the model parameters, i.e. effective temperature, surface gravity and microturbulence. However, in the analysis of a spectroscopic binary, it is not possible to avoid the influence of the veiling factors on the error estimates. The veiling factors depend on the effective temperatures and surface gravities of both stars. Therefore, in order to estimate the sensitivity of an element's abundance to a given stellar parameter, one should also estimate how veiling factors change when one of the stellar parameters of each star is modified. Thus, we also computed $1 \mathrm{D}$ veiling factors for four pairs of models $T_{\mathrm{eff}, \mathrm{A}} / \log g_{\mathrm{A}}, T_{\mathrm{eff}, \mathrm{B}} / \log g_{\mathrm{B}}$ according to the errors of the stellar parameters (see Sect. 5.1), by changing one stellar parameter and fixing the three remaining parameters. The uncertainties on the elemental abundances due to the errors of the different model parameters are listed in Table 4 . The uncertainty of the microturbulence was assumed to be $0.5 \mathrm{~km} \mathrm{~s}^{-1}$.

The errors computed from the dispersion of the line measurements and the signal-to-noise ratios are listed in Table 3. We used the Cayrel formula (Cayrel 1988) to estimate the errors of the observed EWs. Due to the high $\mathrm{S} / \mathrm{N}$ of the spectra we obtained, these errors are typically $\lesssim 0.1 \mathrm{pm}$ in most of the spectral region covered, except for the blue spectrum at $310.0-320.0 \mathrm{~nm}$ where the $\mathrm{S} / \mathrm{N}$ ratio drops significantly. Thus, the dispersion of the measurements for elements with lines in this range is larger than for lines above $400 \mathrm{~nm}$. In Table 3, we give the larger of these two estimates for each element in each star. For Li, the error due to the uncertainty in the continuum level was computed 
Table 4. Abundance errors in CS 22876-032. $\Delta_{T_{\mathrm{eff}}}, \Delta_{\log g}$, and $\Delta_{\xi}$ are the abundance changes caused by changes in $T_{\mathrm{eff}}$ of $100 \mathrm{~K}$ (A) or $150 \mathrm{~K}$ (B), in $\log g$ by $0.1 \mathrm{dex}$, and by $0.5 \mathrm{~km} \mathrm{~s}^{-1}$ in the microturbulence velocity $\xi$. Other column headings and comments as in Table 3 .

\begin{tabular}{|c|c|c|c|c|c|c|c|c|c|c|c|}
\hline Specie & $\log \epsilon(\mathrm{X})_{\odot}$ & {$[\mathrm{X} / \mathrm{H}]_{\mathrm{A}}$} & $\Delta_{\mathrm{A}, T_{\mathrm{eff}}}$ & $\Delta_{\mathrm{A}, \log g}$ & $\Delta_{\mathrm{A}, \xi}$ & $n_{\mathrm{A}}$ & {$[\mathrm{X} / \mathrm{H}]_{\mathrm{B}}$} & $\Delta_{\mathrm{B}, T_{\mathrm{eff}}}$ & $\Delta_{\mathrm{B}, \log g}$ & $\Delta_{\mathrm{B}, \xi}$ & $n_{\mathrm{B}}$ \\
\hline Li I & - & 2.22 & 0.06 & 0 & 0 & 670.8 & 1.75 & 0.08 & 0 & 0 & 670.8 \\
\hline $\mathrm{O}(\mathrm{OH})$ & 8.72 & -1.52 & 0.13 & -0.04 & 0.01 & 4 & -1.75 & 0.23 & 0.02 & 0.04 & 9 \\
\hline $\mathrm{NaI}$ & 6.33 & -3.69 & 0.05 & 0 & -0.01 & 2 & -3.79 & 0.07 & 0 & -0.01 & 589.5 \\
\hline Mg I & 7.58 & -3.11 & 0.04 & 0 & -0.05 & 9 & -3.14 & 0.04 & 0 & -0.04 & 9 \\
\hline Al I & 6.47 & -3.89 & 0.06 & 0 & -0.01 & 2 & -3.75 & 0.06 & 0.02 & -0.03 & 2 \\
\hline Si I & 7.55 & -3.75 & 0.06 & 0.01 & -0.01 & 390.5 & -3.48 & 0.04 & 0.02 & -0.05 & 390.5 \\
\hline $\mathrm{Ca} \mathrm{I}$ & 6.36 & -3.65 & 0.06 & 0 & -0.06 & 422.6 & -3.68 & 0.04 & 0.01 & -0.11 & 422.6 \\
\hline Ca II & 6.36 & -3.68 & 0.01 & 0.03 & -0.06 & 3 & -3.81 & -0.05 & 0.05 & -0.06 & 2 \\
\hline Sc II & 3.17 & -3.73 & 0.04 & 0.03 & -0.01 & 2 & -3.57 & 0.04 & 0.05 & -0.01 & 2 \\
\hline Ti II & 5.02 & -3.25 & 0.05 & 0.03 & -0.04 & 19 & -3.45 & 0.01 & 0.05 & -0.09 & 12 \\
\hline CrI & 5.67 & -3.79 & 0.08 & 0 & -0.01 & 5 & -3.92 & 0.10 & 0.02 & -0.02 & 4 \\
\hline Cr II & 5.67 & -3.38 & 0.03 & 0.03 & -0.07 & 313.2 & -3.30 & -0.05 & 0.05 & -0.08 & 313.2 \\
\hline Mn II & 5.39 & -4.01 & 0.04 & 0.03 & -0.01 & 344.1 & -3.86 & -0.14 & 0.04 & -0.01 & 344.1 \\
\hline $\mathrm{Fe} I$ & 7.50 & -3.66 & 0.09 & -0.01 & -0.05 & 38 & -3.57 & 0.06 & -0.02 & -0.12 & 37 \\
\hline Fe II & 7.50 & -3.51 & 0.03 & 0.02 & -0.02 & 13 & -3.27 & -0.02 & 0.03 & -0.06 & 9 \\
\hline CoI & 4.92 & -2.91 & 0.09 & 0 & -0.01 & 7 & -2.92 & 0.09 & 0.01 & -0.07 & 4 \\
\hline $\mathrm{Ni}$ I & 6.25 & -3.49 & 0.09 & 0 & -0.05 & 12 & -3.45 & 0.07 & 0.01 & -0.13 & 12 \\
\hline
\end{tabular}

from a Monte Carlo simulation, by injecting noise corresponding to the actual $\mathrm{S} / \mathrm{N}$ ratio near the Li line in the best-fit synthetic spectrum. In each case, the $\mathrm{S} / \mathrm{N}$ ratio was estimated taking into account the corresponding veiling factors.

\subsubsection{The iron abundance}

We made a careful selection of 38 reliable Fe I lines in star A and 37 in $\mathrm{B}$, taking into account the radial velocity separation of the two sets of lines in the double-lined spectrum. It is reassuring that we find the same $[\mathrm{Fe} / \mathrm{H}]$ for both stars, within the errors. Note that in our analysis, in contrast with Norris et al. (2000), this was not imposed a priori, and thus supports our determination of atmospheric parameters. Our value of $[\mathrm{Fe} / \mathrm{H}]$ is very close to that by Norris et al. (2000) despite the very different effective temperatures adopted. This results from the fact that different effective temperatures also imply different veiling factors, which must be factored in.

Reviewing the $\left(\langle 3 \mathrm{D}\rangle-1 \mathrm{D}_{\text {MARCS }}\right)$ corrections, we see that $[\mathrm{Fe} / \mathrm{H}]$ is reduced in $3 \mathrm{D}$, and the difference between the two stars increases. We expect the true 3D corrections of the primary star to be largely due to horizontal temperature fluctuations, therefore probably larger than our $\left(\langle 3 \mathrm{D}\rangle-1 \mathrm{D}_{\text {MARCS }}\right)$ correction, and we predict that a full 3D-LTE synthesis will not improve the agreement of $[\mathrm{Fe} / \mathrm{H}]$ between the two stars. Our best estimate of $[\mathrm{Fe} / \mathrm{H}]$ of the system is still the $\left(\langle 3 \mathrm{D}\rangle-1 \mathrm{D}_{\text {MARCS }}\right)$ corrected value for star $\mathrm{A}:[\mathrm{Fe} / \mathrm{H}]=-3.78$, which might be further reduced by a full 3D-LTE synthesis. This confirms that the stars in CS 22876-032 are the most metal-poor dwarfs known to date.

We note that ionisation equilibrium is not achieved in either star. In both, the abundance derived from the Fe II lines is larger, for star B by a factor of two. We note, however, that the Fe II abundance shows a very large scatter in both A and B $(0.25$ and 0.31 dex, respectively) so that, within errors, the Fe I and Fe II abundances remain compatible. The number of Fe II lines measured is very large for stars of this metallicity (13 in star A, 9 in B). However, all the Fe II lines are weak and the majority of them are in the UV range, where the $\mathrm{S} / \mathrm{N}$ ratio of our spectra drops dramatically.

The $\left(\langle 3 \mathrm{D}\rangle-1 \mathrm{D}_{\mathrm{MARCS}}\right)$ corrections for Fe II are in the opposite direction to those for Fe I, making the ionisation imbalance
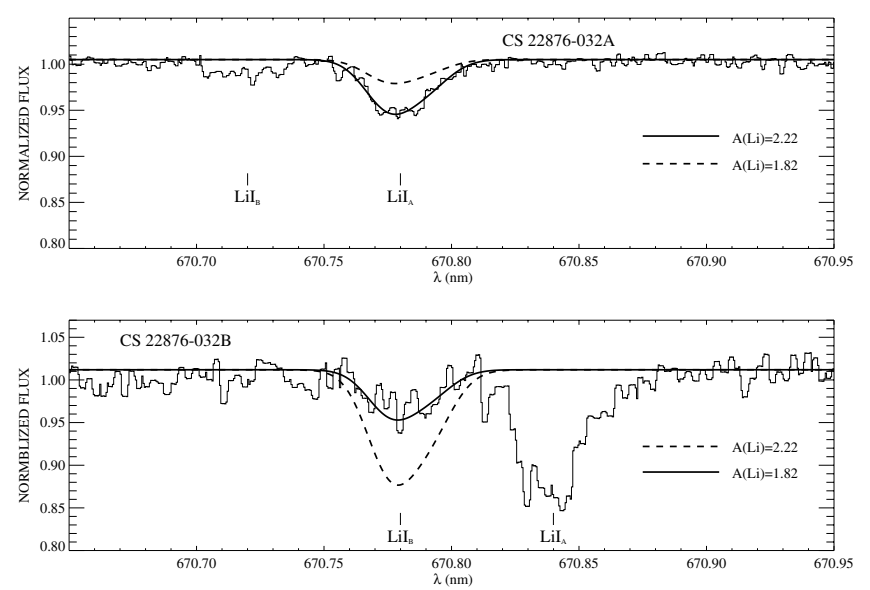

Fig. 7. Synthetic spectral fits to the Li line in the co-added UVES spectrum of star A (top) and B (bottom). The observed spectra have been corrected for veiling (see Table 5), so the lines appear with their intrinsic strength in each star.

worse. The different signs of the $\left(\langle 3 \mathrm{D}\rangle-1 \mathrm{D}_{\mathrm{MARCS}}\right)$ corrections for neutral and ionised species are due to the different ionisation structure of the $\langle 3 \mathrm{D}\rangle$ and $1 \mathrm{D}$ models. We note that for the metal-poor subgiant HD 140283, Shchukina et al. (2005) have performed NLTE computations for Fe I and Fe II, using a single snapshot of a hydrodynamical simulation, and found 3D-NLTE corrections of +0.6 for Fe I and +0.4 for Fe II. To the extent that these computations can be considered representative of the stars in CS 22876-032, we expect that a full 3D-NLTE analysis might achieve a better ionisation balance for iron.

\subsubsection{Lithium}

The high quality of our spectra allowed us to measure the Li doublet in both components of CS 22876-032 for the first time. Figure 7 shows our mean spectra of the Li region, where our well-resolved spectra (only) have been co-aligned on the lines of star A (top) and B (bottom), respectively. The superposed lines of the other star appear only slightly diffuse, because the velocity difference between the two stars is nearly the same in all our 
Table 5. Li abundances in CS 22876-032. $A(\mathrm{Li})_{1 \mathrm{D}, \mathrm{NLTE}, \mathrm{DC}}$ includes corrections for depletion by diffusion (see text).

\begin{tabular}{lrrrrrrr}
\hline \hline Component & $E W_{\text {obs }}(\mathrm{pm})$ & $f_{6708,1 \mathrm{D}}$ & $A(\mathrm{Li})_{1 \mathrm{D}}$ & $A(\mathrm{Li})_{1 \mathrm{D}, \mathrm{NLTE}}$ & $A(\mathrm{Li})_{1 \mathrm{D}, \mathrm{NLTE}, \mathrm{DC}}$ & $\Delta_{\text {3D- 3(D) }}$ & $\Delta_{\text {3D-1D }}$ \\
\hline Star A & 1.32 & 1.36 & 2.22 & 2.18 & 2.18 & -0.17 & -0.19 \\
Star B & 0.42 & 3.74 & 1.75 & 1.77 & 1.84 & -0.02 & -0.29 \\
\hline
\end{tabular}

UVES spectra (see Table 1). The spectra in Fig. 7 have been corrected for veiling, as discussed in Sect. 5.2.1.

The Li abundance of each star was computed from two sets of spectra taken on different nights. We found differences of 0.01 and 0.05 dex between star $\mathrm{A}$ and $\mathrm{B}$ and adopted the average value of the two measurements. Table 5 lists the observed equivalent widths and the average $\mathrm{Li}$ abundances, with and without the NLTE corrections obtained from the tables of Carlsson et al. (1994). We further correct for the effect of depletion as predicted by the standard isochrones of Deliyannis et al. (1990). The correction is negligible for the hot primary, but larger for the secondary (see Table 5).

It is certainly surprising that the two components appear to have a different lithium content. The Li abundance of the primary component seems to be consistent with that observed in other metal-poor stars, i.e., the Spite plateau (Spite \& Spite 1982b,a; Bonifacio \& Molaro 1997; Ryan et al. 1999; Meléndez \& Ramírez 2004; Charbonnel \& Primas 2005; Asplund et al. 2006; Bonifacio et al. 2007), in spite of the uncertainties on the temperature scales adopted over the years by the various authors, but the secondary star definitely seems to exhibit a lower Li content.

According to the Li depletion isochrones of Deliyannis et al. (1990), if star B were $350 \mathrm{~K}$ cooler than we assume, the correction for $A(\mathrm{Li})$ would be 0.6 dex. Such a change in $T_{\text {eff }}$ would also imply a slightly higher $\log g$ from the isochrone, so the model dependencies given in Table 4 would imply that $A(\mathrm{Li})$ should be reduced by 0.17 , giving a "corrected" $\mathrm{Li}$ abundance of $A(\mathrm{Li})=2.20$, in essential agreement with $A(\mathrm{Li})$ of the primary (we do not consider variations in the temperature of star A). By changing simultaneously the effective temperatures of the pair (within the range allowed by photometry), we would derive different $\mathrm{Li}$ abundances for each of the components. However, we would also change the veiling factors, and the final abundance difference between the two stars would be roughly the same.

We have checked whether inaccurate veiling corrections, especially for the fainter lines of star B, could be the cause of the different $\mathrm{Li}$ abundances. It turns out that this is impossible: bringing $A(\mathrm{Li})_{\mathrm{B}}$ into consistency with the Spite plateau would require a doubling of the veiling correction at $670 \mathrm{~nm}$; given that we find consistent abundances for $\mathrm{Na}$ at $590 \mathrm{~nm}$ and $\mathrm{Mg} \mathrm{I}$ at $880 \mathrm{~nm}$, the veiling correction cannot be off by a factor two at the intermediate wavelength.

For the Li lines we also performed a full 3D-LTE synthesis using Linfor3D. The resulting corrections are listed in Table 5. Since the 3D computation was performed in LTE, the Li abundances must not be taken as definitive, as shown by Cayrel \& Steffen (2000) and Asplund et al. (2003). It is, however, interesting to notice that while the $3 \mathrm{D}$ effect in star A is almost entirely due to the horizontal temperature fluctuations, for star B it is almost entirely due to the cooler average temperature profile of the 3D model. A full 3D-NLTE synthesis of Li in CS 22876-032 is beyond the scope of this paper. Full 3D-NLTE synthesis of the $\mathrm{Li}$ profile in HD74000 was recently addressed by Cayrel et al. (2007).
Table 6. 3D abundance corrections for the $\mathrm{OH}$ lines. $[\mathrm{O} / \mathrm{H}]$ and $[\mathrm{O} / \mathrm{Fe}]$ are relative to $\log \epsilon(\mathrm{O})_{\odot}=8.72$.

\begin{tabular}{ccccc}
\hline \hline$\lambda(\mathrm{nm})$ & {$[\mathrm{O} / \mathrm{H}]_{1 \mathrm{D}}$} & {$[\mathrm{O} / \mathrm{Fe}]_{1 \mathrm{D}}$} & $\Delta_{3 \mathrm{D}-\langle 3 \mathrm{D}\rangle}$ & $\Delta_{3 \mathrm{D}-1 \mathrm{D}}$ \\
\hline \multicolumn{5}{c}{$\mathrm{CS} 22876-032 \mathrm{~A}$} \\
\hline 311.21 & -1.48 & 2.18 & -0.633 & -1.546 \\
311.47 & -1.55 & 2.11 & -0.536 & -1.328 \\
312.26 & -1.56 & 2.10 & -0.590 & -1.420 \\
313.43 & -1.48 & 2.18 & -0.797 & -1.664 \\
\hline \multicolumn{5}{c}{$\mathrm{CS} 22876-032 \mathrm{~B}$} \\
\hline 312.26 & -1.63 & 1.94 & 0.027 & -0.985 \\
312.77 & -1.85 & 1.72 & 0.014 & -0.868 \\
312.83 & -1.68 & 1.89 & 0.033 & -1.009 \\
313.03 & -1.94 & 1.63 & -0.004 & -1.045 \\
313.32 & -1.69 & 1.88 & 0.003 & -0.856 \\
313.66 & -1.75 & 1.82 & -0.015 & -0.849 \\
314.30 & -1.80 & 1.77 & -0.011 & -0.815 \\
317.45 & -1.75 & 1.82 & -0.017 & -0.916 \\
317.77 & -1.70 & 1.87 & -0.009 & -0.906 \\
\hline
\end{tabular}

\subsubsection{Oxygen}

The oxygen abundances have been derived from UV OH lines of the $(0-0)$ vibrational band of the $A^{2}$ Sect.igma $-X^{2} \Pi$ electronic system. The use of these lines for oxygen measurements in metal-poor stars was pioneered by Bessell et al. (1991). We were able to measure four lines in the primary and nine lines in the secondary. Following the extensive surveys of $\mathrm{OH}$ lines in metal-poor stars by Israelian et al. (1998); Boesgaard et al. (1999b); Israelian et al. (2001), and the controversial finding of strongly increasing $[\mathrm{O} / \mathrm{Fe}]$ with decreasing metallicity, Asplund \& García Pérez (2001) warned of the possible role of 3D effects on the formation of these lines. For this reason we decided to compute ad hoc $\mathrm{CO}^{5} \mathrm{BOLD}$ hydrodynamical simulations for this system to correctly evaluate the 3D effects. Our results are summarised in Table 6. Some of the analysed lines are shown in Figs. 8-10.

The 3D effects are clearly large and considerably different between the two stars. Asplund \& García Pérez (2001) attributed the $3 \mathrm{D}$ corrections primarily to the different average temperature profile of the 3D models, in particular to their extremely cool outer layers. However, we see that for the primary star the $3 \mathrm{D}$ correction is almost evenly shared between average temperature profile and horizontal temperature fluctuations ${ }^{4}$.

Our cooler 3D model atmosphere exhibits much smaller temperature fluctuations around the mean than the hotter one. In the cool component the average temperature becomes so low that a substantial amount of $\mathrm{H}_{2}$ is formed in the higher photospheric layers. The associated increase of the specific heat makes it much harder for pressure fluctuations to introduce temperature fluctuations. Due to the smaller temperature fluctuations the cooler $3 \mathrm{D}$ and $\langle 3 \mathrm{D}\rangle$ models provide essentially the same abundances. The model of the primary star is hotter on average, $\mathrm{H}_{2}$ molecules are much less abundant, and temperature

\footnotetext{
$4-0.64$ dex due to temperature fluctuations and -0.85 dex due to average temperature profile.
} 

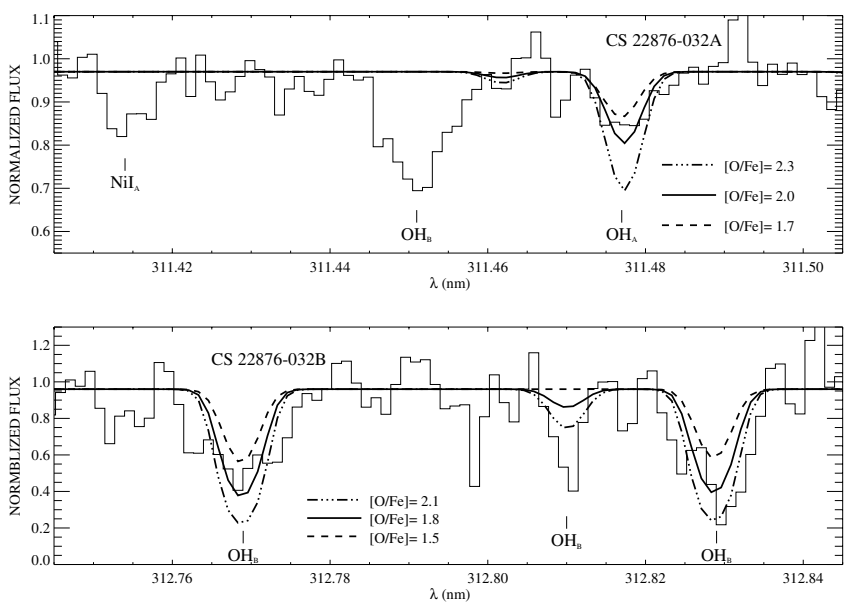

Fig. 8. Synthetic spectral fits to $\mathrm{OH}$ lines in the co-added UVES spectrum of star A (top) and B (bottom) in CS 22876-032. The observed spectra have been corrected for veiling factors of 1.30 (A) and 4.36 (B).
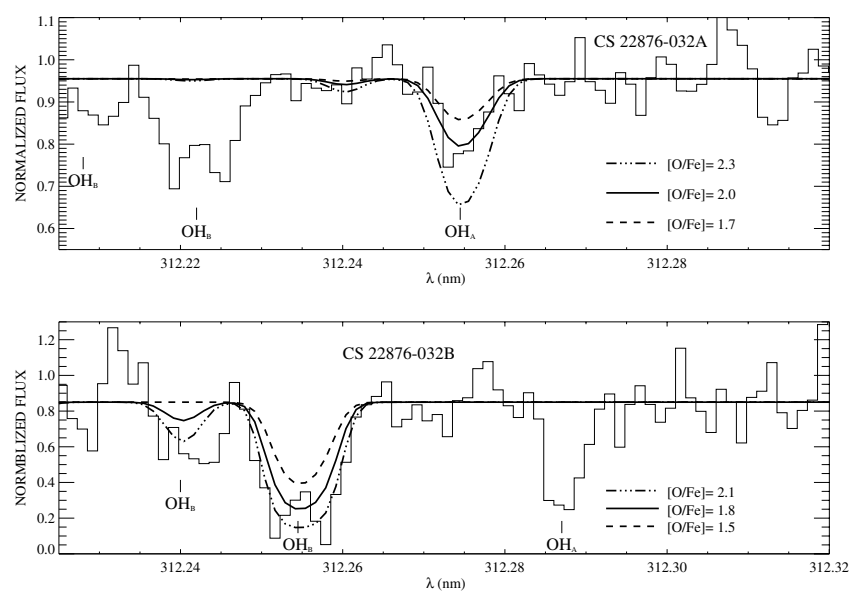

Fig. 9. Same as Fig. 8 for another $\mathrm{OH}$ line.
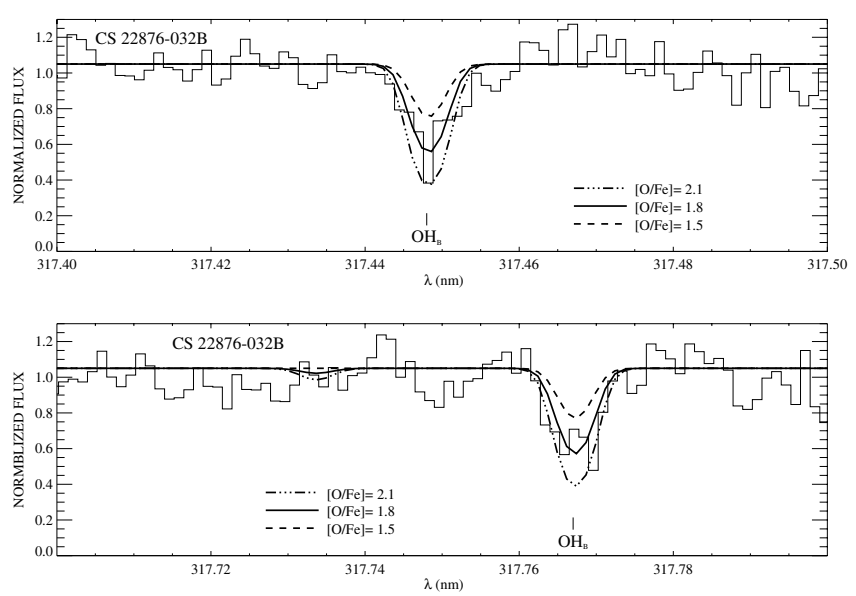

Fig. 10. Synthetic spectral fits to OH lines in the co-added UVES spectrum of star B. The observed spectra have been corrected for a veiling factor of 4.36 .

fluctuations are much more pronounced. Consequently, the resulting abundances differ between $3 \mathrm{D}$ and $\langle 3 \mathrm{D}\rangle$ models.

Figure 11 illustrates the situation. The 3D model exhibits a stronger cooling with respect to the $1 \mathrm{D}_{\mathrm{LHD}}$ model at $5900 \mathrm{~K}$ than is the case at $6500 \mathrm{~K}$. Over a wide pressure range, the structure is almost adiabatic and passes through a region of substantial

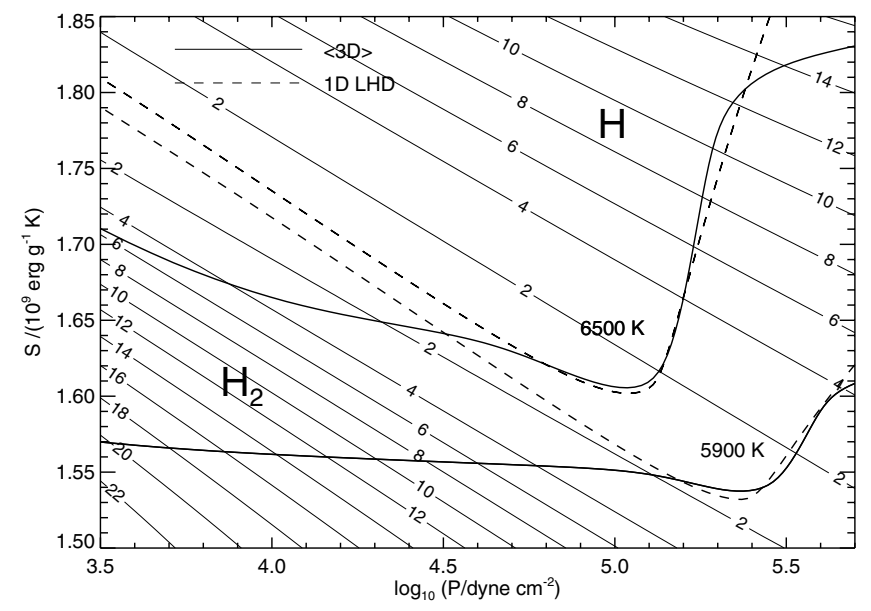

Fig. 11. Entropy profiles of the $\langle 3 \mathrm{D}\rangle \mathrm{CO}^{5} \mathrm{BOLD}$ model atmospheres (solid lines), compared to $1 \mathrm{D}_{\mathrm{LHD}}$ models (dashed) for stars B $(5900 \mathrm{~K})$ and $\mathrm{A}(6500 \mathrm{~K})$. The labelled contours indicate the specific heat at constant pressure in units of $10^{8} \mathrm{erg} \mathrm{g}^{-1} \mathrm{~K}^{-1}$ (see text).

$\mathrm{H}_{2}$ molecule formation, indicated by the rather high values of the specific heat, suppressing temperature fluctuations in that region. The almost adiabatic structure of the cool model also indicates that the convective overshooting is very efficient compared to radiative heating, and has driven the thermal structure into almost adiabatic equilibrium.

The large difference in the behaviour of our two models warns us that to measure reliable abundances from $\mathrm{OH}$ one needs a grid of 3D models which is fairly dense in temperature, to capture, for any metallicity, the $T_{\mathrm{eff}}$ at which $\mathrm{H}_{2}$ formation sets in.

Finally, we note that the $\mathrm{O}$ abundance derived from $\mathrm{OH}$ lines is rather sensitive to the surface gravity, as can be seen from Table 7. An increase of gravity of 0.3 dex introduces a decrease in $\mathrm{O}$ abundance of about 0.1 dex. This gravity dependence is larger than that of Fe I, however it is advisable to use Fe I to derive $[\mathrm{O} / \mathrm{Fe}]$, since the gravity dependence of $\mathrm{Fe}$ II is in the opposite direction. Note that the values reported in Table 7 have been estimated without revising the veiling factor when changing the surface gravity of the models, contrary to the error estimates described in Sect. 5.2.2.

\subsubsection{Other elements}

The Be II resonance doublet at $313.0 \mathrm{~nm}$ is within the wavelength range covered by our spectra. The $\mathrm{S} / \mathrm{N}$ ratio in that region is $\sim 25$, yet none of the Be lines are detected, which implies an upper limit for the $\mathrm{Be}$ abundance $\log (\mathrm{Be} / \mathrm{H})<-13.0$. As this is an order of magnitude higher than the Be abundance expected for these stars from the trend of Be abundance with metallicity (Gilmore et al. 1992; Ryan et al. 1992; Molaro et al. 1997; Boesgaard et al. 1999a; Primas 2002; Boesgaard \& Novicki 2006), this result is not significant.

For the other elements, abundances were determined directly from the $E W \mathrm{~s}$, except for Sc and Co for which we used spectrum synthesis to take hyperfine splitting (HFS) into account. For Co we used the $A$ and $B$ factors measured by Pickering (1996). For the Sc II $361.3 \mathrm{~nm}$ line we used the $A$ and $B$ factors measured by Gangrsky et al. (2006). In both cases we used the code LINESTRUC of Wahlgren (2005) to compute HFS components. For the Sc II $424.6 \mathrm{~nm}$ line we used the HFS components given in Table 5 of McWilliam et al. (1995). The detailed atomic data of HFS for Sc and Co are provided in Table A.2. For Mn we 
Table 7. 1D gravity effects on the $\mathrm{Fe}$ and $\mathrm{O}$ abundances.

\begin{tabular}{lrrr}
\hline \hline Species & $\Delta \log g$ & $\Delta[\mathrm{X} / \mathrm{H}]_{\mathrm{A}}$ & $\Delta[\mathrm{X} / \mathrm{H}]_{\mathrm{B}}$ \\
\hline $\mathrm{OH}$ & 0.3 & -0.12 & -0.08 \\
$\mathrm{Fe}$ I & 0.3 & -0.01 & -0.02 \\
$\mathrm{Fe}$ II & 0.3 & 0.09 & 0.11 \\
\hline
\end{tabular}

used a single line, the strongest of the Mn II lines of Mult. 3, for which HFS is negligible according to Castelli \& Hubrig (2004).

Silicon was only measured from the Si I line at $390.5 \mathrm{~nm}$. In metal-poor cool giants, the silicon abundance is derived from a line at $410.3 \mathrm{~nm}$, since the line Si I $390.5 \mathrm{~nm}$ is severely blended with $\mathrm{CH}$ lines (Cayrel et al. 2004). The Si I at $410.3 \mathrm{~nm}$ is very weak in metal-poor dwarfs, but $\mathrm{CH}$ lines are so weak that the line at $390.5 \mathrm{~nm}$ can be used.

Aluminium was measured from the resonance lines Al I $394.4 \mathrm{~nm}$ and Al I $396.1 \mathrm{~nm}$. The Al I $394.4 \mathrm{~nm}$ is blended with $\mathrm{CH}$ lines, which are also extremely weak in metal-poor dwarfs and were not taken into account. We computed synthetic spectra of the $\mathrm{CH}$ lines at $390.5 \mathrm{~nm}$ and $394.4 \mathrm{~nm}$ for the stellar parameters of both dwarfs, and the $\mathrm{CH}$ lines are not visible even for $[\mathrm{C} / \mathrm{Fe}]$ as large as +2 dex. However, the $394.4 \mathrm{~nm}$ line provided a significantly larger abundance than that derived from the $396.1 \mathrm{~nm}$ line in both stars. For this reason, in Table 3 we give the average $\mathrm{Al}$ abundance derived from both lines with a large dispersion of $\sim 0.2$ dex.

Using $\langle 3 D\rangle$ models, we determine the $\left(\langle 3 \mathrm{D}\rangle-1 \mathrm{D}_{\text {MARCS }}\right)$ corrections listed in Table 3 . We note that the $\left(\langle 3 D\rangle-1 D_{\text {MARCS }}\right)$ corrections also take into account different veiling factors estimated using $\langle 3 \mathrm{D}\rangle$ models and 1D OSMARCS models. In general, the veiling factors estimated from $\langle 3 \mathrm{D}\rangle$ models are higher for star B and lower for star A than those obtained using 1D models. This effect is especially important for the secondary star, and becomes more significant at shorter wavelengths. Taking into account this effect, it is interesting to note that $\left(\langle 3 \mathrm{D}\rangle-1 \mathrm{D}_{\text {MARCS }}\right)$ corrections are in general negative for neutral species and positive for ionised species, at least for the primary star, for which the veiling factors are not significantly different from those estimated using 1D models, as already noted for iron. This reflects the different ionisation structure of $1 \mathrm{D}$ and $\langle 3 \mathrm{D}\rangle$ models. The $\left(\langle 3 D\rangle-1 D_{\text {MARCS }}\right)$ corrections do not help to achieve ionisation equilibrium for $\mathrm{Ca}, \mathrm{Cr}$ and $\mathrm{Fe}$, the only elements for which abundances were determined from both neutral and ionised species.

\section{Discussion}

\subsection{Lithium}

In Fig. 12 we show the lithium abundances for the two components of CS 22876-032, together with our data from Paper VII, including the data of Asplund et al. (2006) rescaled in $[\mathrm{Fe} / \mathrm{H}]$ and $A(\mathrm{Li})$ to be homogeneous with our own. Star A appears to have a Li abundance at the same level as the majority of stars with metallicity below -2.5 , if anything slightly higher. Star B appears to be far below ${ }^{5}$ any of the other measured stars. It should be noted that all the other stars in Fig. 12 have effective temperatures determined from the wings of $\mathrm{H} \alpha$ using the broadening theory of Barklem et al. (2000), while for CS 22876-032 they have been determined independently from colours and isochrones. However, the reasonable agreement between our computed $\mathrm{H} \alpha$

\footnotetext{
$5-0.33$ dex below the average $\mathrm{Li}$ abundance of the stars presented in Paper VII.
}

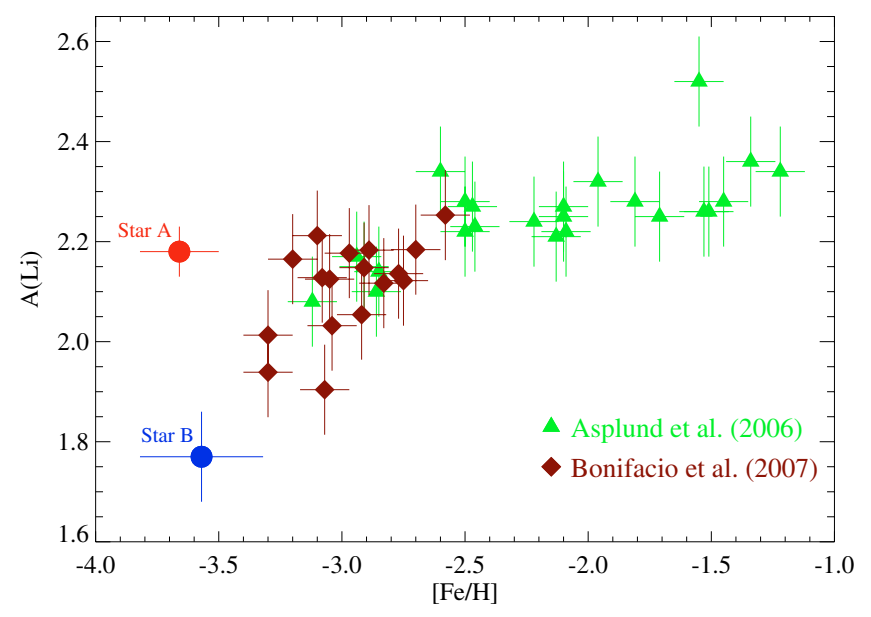

Fig. 12. 1D-NLTE $\mathrm{Li}$ abundances vs. $[\mathrm{Fe} / \mathrm{H}]$ for the stars in CS 22876-032 (circles) and in other metal-poor dwarfs as reported by Asplund et al. (2006, triangles) and Bonifacio et al. (2007, diamonds). The Li abundances by Asplund et al. (2006) were recomputed using TURBOSPECTRUM as reported by Bonifacio et al. (2007). No 3D-LTE or 3D-NLTE was considered.

profile and the observed profile shown in Fig. 6 suggests that the two temperature scales are fairly close.

The fact that at the lowest observable metallicity, star A remains at the level of the plateau suggests that there is no downturn or decrease in $\mathrm{Li}$ abundance at the lowest metallicities. This suggests that the slope of $A(\mathrm{Li})$ with $[\mathrm{Fe} / \mathrm{H}]$ which is detectable in the sample of Asplund et al. (2006) (but not in that of Paper VII alone) is not real, but rather an artifact due to the $\mathrm{H} \alpha$ temperature scale. It is possible that it is ultimately due to our inability to correctly model the atmospheres of extremely metalpoor stars and the wings of Balmer line profiles. In Paper VII we argued that the data could suggest either a vertical drop or an increased scatter in $A(\mathrm{Li})$ at the lowest metallicities. The drop now seems to be ruled out by the $A(\mathrm{Li})$ measured in star A. The measurement in star B, taken at face value, may support the idea that at metallicities below -2.5 , the Spite plateau displays a sizeable scatter.

In Sect. 5.2.4 we pointed out how the difference of the $\mathrm{Li}$ abundance between the two stars could be resolved by assuming that the temperature of star B was $5550 \mathrm{~K}$. Star B would thus be subject to considerable Li depletion, according to the standard Li depletion isochrones of Deliyannis et al. (1990). Such isochrones are available only for metallicities considerably higher than that of CS 22876-032. If, for any reason, either the lower metallicity of our system, or inclusion of other physical phenomena, the dependence of Li depletion on $T_{\text {eff }}$ is steeper than predicted by purely diffusive standard isochrones, then to reconcile the $\mathrm{Li}$ abundances of the two stars, the temperature of star B could be higher than $5550 \mathrm{~K}$. Considering that our estimated error on the effective temperature of star B is $150 \mathrm{~K}(2 \sigma)$, such cooler temperatures are not totally implausible.

In our view, the existence of a real scatter in $\mathrm{Li}$ abundances at the lowest metallicities remains to be established beyond any reasonable doubt. It is, nevertheless, worthwhile discussing the possible implications of such a scatter, if real.

We have already noted in Paper VII that by arbitrarily dividing the sample into two sub-samples, one with metallicity below -3.0 and the other above, the scatter of the "higher" metallicity sample was $0.05 \mathrm{dex}$, while the scatter of the "lower" metallicity sample was 0.11 . If to the lower metallicity sample we add the 


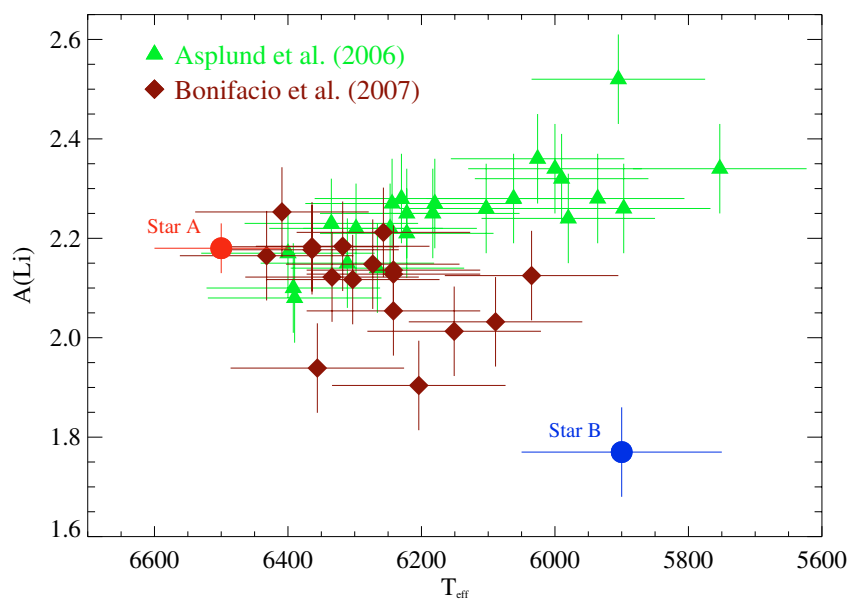

Fig. 13. Same as Fig. 12, but in the Li- $T_{\text {eff }}$ plane.

two stars of CS 22876-032, the scatter increases only slightly, to 0.12 dex. Richard et al. (2005) have studied the effect of atomic diffusion in the presence of turbulence and concluded that the observations of the Spite plateau could be explained by starting from a primordial $A(\mathrm{Li})$, compatible with the baryonic density derived from the WMAP experiment. The effect of atomic diffusion, countered by a suitably parametrised turbulence, can explain the present level and low scatter of the Spite plateau.

From their $\mathrm{Li}$ isochrones in the presence of pure atomic diffusion (Fig. 5 of Richard et al. 2005) it is obvious that one should expect $\mathrm{Li}$ abundances in the range $1.70 \leq A(\mathrm{Li}) \leq 2.35$. The sample constituted by the 8 stars from Paper VII with metallicity below -3.0 and the two stars in CS 22876-032 spans the range $1.91-2.20$ in $A(\mathrm{Li})$. One could therefore suspect that in extremely metal-poor stars, turbulence is lower and atomic diffusion more efficient, causing the increased scatter in Li abundance. In Fig. 13 we see no clear trend of $A(\mathrm{Li})$ vs. $T_{\text {eff. }}$. When comparing this figure with Fig. 5 of Richard et al. (2005) we note that the "high" Li abundances of the hottest stars in the sample (among which is the primary of CS 22876-032), preclude any clear resemblance between the two pictures. Therefore, current pure diffusion models are unable to explain the behaviour of $\mathrm{Li}$ abundances with $[\mathrm{Fe} / \mathrm{H}]$ and $T_{\text {eff. }}$. It is possible, however, that they may do so, after an ad hoc parametrisation of turbulence with metallicity.

Recently Korn et al. (2006, 2007) found that the models of Richard et al. (2005), with a suitable value for the turbulent diffusion coefficient, can explain the 0.12 dex difference in $A(\mathrm{Li})$ they find between stars at the turn-off and on the subgiant branch stars of the globular cluster NGC 6397. The same authors, however point out that assigning temperatures for the TO stars, hotter by $170 \mathrm{~K}$ (therefore close to the temperatures adopted by Bonifacio et al. 2002, for the TO stars of this cluster), such a difference would vanish.

Though suggestive, the applicability of such turbulent diffusive models remains to be proven. The main cause of concern is the parametrisation of the turbulent diffusive coefficient, which is linked to a fixed temperature, and not to the bottom of the convective zone (Richard et al. 2002).

In order to strengthen the observational constraints on such models and refine the estimates of the scatter and slope (or lack thereof) of the extreme metal-poor end of the Spite Plateau, further high-quality spectroscopy of EMP stars and additional accurate constraints on the effective temperatures of the whole sample are highly desirable.

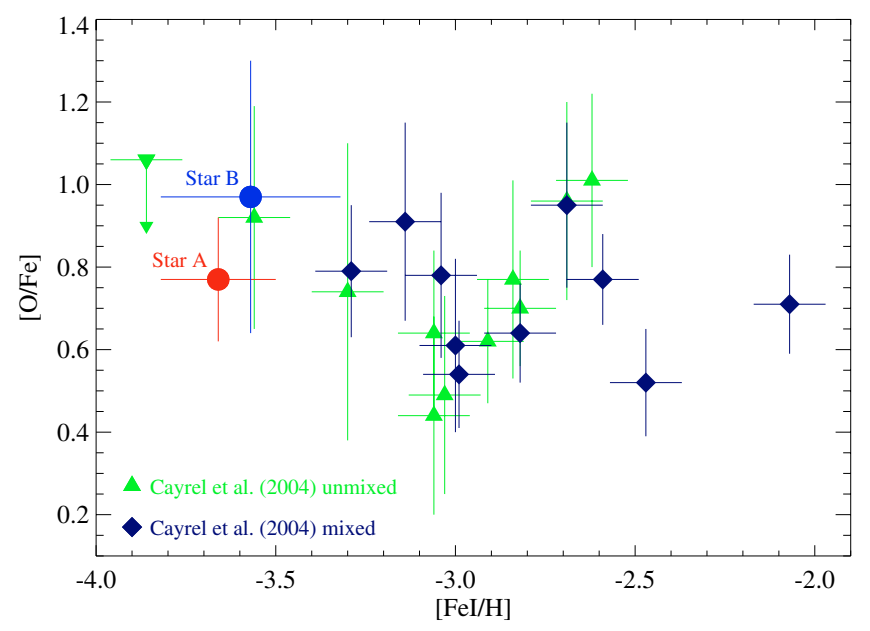

Fig. 14. 3D $[\mathrm{O} / \mathrm{Fe}]$ ratios of the stars in CS 22876-032 (circles) and in the metal-poor giants of Paper V. Triangles: "unmixed", diamonds "mixed" stars; downward triangle with arrow: upper limit for CS 22172-002.

\subsection{Oxygen}

Figure 14 compares our 3D-LTE O abundances obtained from OH UV bands in CS 22876-032 with the high-quality measurements of Paper V for giant stars (mixed and unmixed), obtained from the [OI] $630 \mathrm{~nm}$ line. We note that we computed the $3 \mathrm{D}-\mathrm{LTE}[\mathrm{O} / \mathrm{Fe}]$ ratios using the true 3D-LTE oxygen abundances and the $\langle 3 \mathrm{D}\rangle$ Fe I abundances. We chose Fe I rather than Fe II abundances as the reference, because their sensitivity to the surface gravity is similar to that of the $\mathrm{OH}$ bands. The reader might wonder why we find different $\mathrm{O}$ abundances for stars $\mathrm{A}$ and B, but they are in fact consistent within the error bars, which mainly reflect the uncertainties in the effective temperature (see Table 4).

The abundances derived from [OI] are the raw values obtained from 1D-LTE model atmospheres. One worry is the possible effects of granulation on the abundances derived from the [OI] line in giants. In a recent paper, Collet et al. (2007) investigated the 3D effects in giant stars and found very small corrections for the $[\mathrm{OI}]$ lines for metallicities down to -2.0 , but sizeable (almost 0.2 dex) downward corrections for $[\mathrm{O} / \mathrm{Fe}]$ ) at metallicity -3.0 (their Fig. 13 and Table 3). If we apply the corrections interpolated and extrapolated from Table 3 of Collet et al. (2007) to the giants of Paper $\mathrm{V}$, the $[\mathrm{O} / \mathrm{Fe}]$ decreases in all the giants and the good agreement between CS 22876-032 and the giants no longer holds. The mean $[\mathrm{O} / \mathrm{Fe}]$ of the giants would be 0.51 , while that of CS 22876-032 is 0.87. If instead we take the measurements of Paper V at face value, the mean $[\mathrm{O} / \mathrm{Fe}]$ is 0.72 .

At present we have no full 3D models for giant stars, however, from a few snapshots for models of $T_{\text {eff }}=4900, \log g=2.0$ and metallicity -2.0 and -3.0 we find little difference at the two metallicities, and very small $3 \mathrm{D}$ corrections. The $3 \mathrm{D}-1 \mathrm{D}_{\mathrm{LHD}}$ abundance correction amounts to -0.006 dex at metallicity -2.0 and -0.037 dex at -3.0 , which is negligible. For our $\mathrm{CO}^{5} \mathrm{BOLD}$ models of giants the difference between the mean 3D temperature structure and a corresponding $1 \mathrm{D}_{\mathrm{LHD}}\left(\alpha_{\mathrm{MLT}}=1.0\right)$ model is not large. In particular we do not find the substantial cooling of the highest photospheric layers at metallicity -3.0 , which Collet et al. (2007) find (see their Fig. 1). Moreover, the mean $3 \mathrm{D}$ temperature profile is slightly hotter than the $1 \mathrm{D}_{\mathrm{LHD}}$ temperature profile in the [OI] $630 \mathrm{~nm}$ line-forming layers around $\log \tau \sim-1$. The differences between our assessment of the 
impact of granulation on the abundances derived from the [OI] $630 \mathrm{~nm}$ line in giants, and that of Collet et al. (2007), will be further investigated in the future. This might be rooted in the different binning schemes adopted by the two codes for the opacity (4 opacity bins for the Stein \& Nordlund code and 6 opacity bins for $\left.\mathrm{CO}^{5} \mathrm{BOLD}\right)$. In any case we believe that, at present, it is safer not to apply any $3 \mathrm{D}$ correction to the $\mathrm{O}$ abundances derived for giants from the [OI] $630 \mathrm{~nm}$ line.

In Paper $\mathrm{V}$ we found that the mean value of $[\mathrm{O} / \mathrm{Fe}]$ in the range $-3.6<[\mathrm{Fe} / \mathrm{H}]<-2.5$ was about $0.72 \pm 0.17$. The weighted average value in $\mathrm{CS} 22876-032,[\mathrm{O} / \mathrm{Fe}]=0.83 \pm 0.15$, agrees with this determination. This mean value does not exclude a slight increase of the ratio $[\mathrm{O} / \mathrm{Fe}]$ in the range $-3.6<$ $[\mathrm{Fe} / \mathrm{H}]<-2.5$ as seen in the theoretical predictions of the chemical evolution models presented by François et al. (2004). A more detailed discussion of the general behaviour of oxygen with metallicity will be made in forthcoming investigations, hopefully after 3D corrections will be determined for all the stars, and for different lines of oxygen, Fe I and Fe II.

\subsection{The Odd-Z light elements}

The LTE $[\mathrm{Na} / \mathrm{Fe}]$ abundance ratios of both stars appear consistent with the Galactic trend of this element in EMP giants (Cayrel et al. 2004), subgiants and turn-off stars of similar metallicity (Andrievsky et al. 2007). NLTE corrections are expected to be larger for the metal-poor giants than for dwarfs. We have estimated the NLTE corrections ${ }^{6}$ for Na to be $\Delta_{\text {NLTE }} \lesssim-0.06$ dex, according to the NLTE corrections reported in Table 2 of Andrievsky et al. (2007). After applying these corrections, Na abundances of the CS 22876-032 dwarfs remain compatible with those of metal-poor giants and dwarfs, which exhibit almost a constant ratio $[\mathrm{Na} / \mathrm{Fe}] \sim-0.20$ in the metallicity range $-4.0<[\mathrm{Fe} / \mathrm{H}]<-2.5$.

The LTE $[\mathrm{Al} / \mathrm{Fe}]$ abundance ratios of the dwarfs in this system are $\sim 0.5$ dex larger than those in metal-poor giants with similar iron content. However, aluminium is also expected to exhibit significant NLTE corrections (Baumüller \& Gehren 1997), which might explain this difference, as was the case for $\mathrm{Na}$. This element also exhibits an almost constant ratio $[\mathrm{Al} / \mathrm{Fe}] \sim-0.10$ for giants in the metallicity range $-4.0<[\mathrm{Fe} / \mathrm{H}]<-2.5$ when a fixed NLTE correction of +0.65 dex is considered.

Within the errors, $[\mathrm{Sc} / \mathrm{Fe}]$ is consistent with, although $0.2-0.3$ dex lower than, the $[\mathrm{Sc} / \mathrm{Fe}]$ ratio in EMP giants which shows an almost constant ratio $[\mathrm{Sc} / \mathrm{Fe}] \sim 0$. Note that the $[\mathrm{X} / \mathrm{Fe}]$ ratios given in Table 3 were computed relative to Fe II for ionised species. As noted in Sect. 5.2.3, the Fe II abundances are less reliable than those for Fe I because the Fe II lines in EMP dwarfs are very weak, especially for star B in CS 22876-032. Therefore, $[\mathrm{X} / \mathrm{Fe}]$ for ionised species in star $\mathrm{B}$ should be regarded with caution.

\subsection{The $\alpha$ elements}

The $[\mathrm{Mg} / \mathrm{Fe}]$ ratios in both stars of CS 22876-032 are approximately consistent with those found in EMP giants (Cayrel et al. 2004), subgiants and dwarfs (Cohen et al. (2004), Bonifacio et al. 2007, in prep.), at the level of $\sim 0.3-0.4$ dex.

$[\mathrm{Ca} / \mathrm{Fe}]$ was derived from both $\mathrm{Ca}$ I and $\mathrm{Ca}$ II lines, which yield similar $[\mathrm{Ca} / \mathrm{H}]$ abundances, at least for the primary star. However, although the $[\mathrm{Ca} / \mathrm{Fe}]$ ratios differ by $\sim 0.2$ dex, they

\footnotetext{
${ }^{6} \Delta_{\mathrm{NLTE}}=\log \epsilon(\mathrm{X})_{\mathrm{NLTE}}-\log \epsilon(\mathrm{X})_{\mathrm{LTE}}$.
}

seem to be slightly lower than those measured in metal-poor giants and dwarfs, where a constant $[\mathrm{Ca} / \mathrm{Fe}] \sim 0.4$ is seen.

[Si/Fe] also seems to be low, at $[\mathrm{Si} / \mathrm{Fe}] \sim-0.1$ for stars $\mathrm{A}$ and $\sim+0.1$ for the secondary, compared to the constant $[\mathrm{Si} / \mathrm{Fe}] \sim$ 0.4 for metal-poor giants. However, other metal-poor dwarfs show similar abundances, which might be related to the different Si lines used in giants and dwarfs (Bonifacio et al. 2007, in prep.).

Finally, $[\mathrm{Ti} / \mathrm{Fe}]$ is constant at $\sim 0.3$ in EMP giants. While we find a similar result for star $\mathrm{A},[\mathrm{Ti} / \mathrm{Fe}]$ in star $\mathrm{B}$ is completely different due to its high Fe II abundance. We note that the standard deviation in $[\mathrm{Ti} / \mathrm{Fe}]$ is relatively high in both stars $(\sigma \sim 0.3)$, although the mean abundances are better determined when averaging the results from the 19 lines in star A and 12 in B.

\subsection{The iron-peak elements}

Chromium was derived from both $\mathrm{Cr}$ I and $\mathrm{Cr}$ II lines, and we find a difference of $\sim 0.2$ dex for the $[\mathrm{Cr} / \mathrm{Fe}]$ ratios. $[\mathrm{Cr} \mathrm{I} / \mathrm{Fe} \mathrm{I}]$ in CS 22876-032 appears to agree with that in other EMP dwarfs, but is slightly higher than seen in EMP giants.

The ratio $[\mathrm{Mn} / \mathrm{Fe}]$ is consistent with the other EMP giants and dwarfs, at $[\mathrm{Mn} / \mathrm{Fe}] \sim-0.5$. [Ni/Fe] also agrees with the values reported for EMP giants, which show a constant $[\mathrm{Ni} / \mathrm{Fe}] \sim 0$.

Cobalt is found to be slightly enhanced in CS 22876-032 relative to the gradually increasing trend of $[\mathrm{Co} / \mathrm{Fe}]$ with decreasing $[\mathrm{Fe} / \mathrm{H}]$ observed in giants, although marginally compatible within the errors.

\subsection{Could CS 22876-032 be a triple system?}

One could ask whether a third star in CS 22876-032 might contribute significantly to the total light and the veiling of the lines of the two main components we have discussed so far. Such a star would need to have a mass above $\sim 0.5 M_{\odot}$ in order to have any significant effect on the observed spectrum. The presence of such a third star can be ruled out by two independent pieces of evidence.

First, we have individual spectra of CS 22876-032 with S/N ratios $\sim 100$ in the region of the $\mathrm{Mg} \mathrm{I} b$ triplet. These are among the strongest stellar lines seen in these EMP stars and would be at least as strong in the third star. The Cayrel formula predicts that any line with an $E W$ above $\sim 0.15 \mathrm{pm}$ would be detected at the $3 \sigma$ level. Assuming $T_{\text {eff }}=5500 \mathrm{~K}$ and $\log g=4.7$ (from the isochrone) plus $[\mathrm{Fe} / \mathrm{H}]=-3.6$ and $[\mathrm{Mg} / \mathrm{Fe}]=+0.2$ for the hypothetical third star, we need to dilute the strongest line of the $\mathrm{Mg}$ I b triplet at $518.36 \mathrm{~nm}$ which would show an intrinsic $E W$ of $\sim 15.0 \mathrm{pm}$. In Appendix A we define the veiling factors for a triple system and conclude that the non-detection of the $518.36 \mathrm{~nm}$ line requires $f_{3}>100$. We can therefore conclude that any third star contributes negligibly to the total light of CS 22876-032.

A second line of evidence is available from the radial velocities, which are accurate to $\sim 1 \mathrm{~km} \mathrm{~s}^{-1}$ and cover a period of 16 years. Any third star of mass comparable to A and B should leave significant trends in the velocity residuals from the orbital solution for periods shorter than several decades. We have therefore attempted sinusoidal fits to the velocity residuals for both stars and find periods of the order of 1300 days in both cases. First, the standard deviations around these fits are 0.83 and $0.51 \mathrm{~km} \mathrm{~s}^{-1}$, well below the purely observational errors, which shows that these results cannot be statistically significant. Second, a period ratio of $\sim 3$ between the outer and inner orbits 
is far too small for a triple system to be dynamically stable. A white dwarf in an orbit of much longer period is a possibility, but would not be detectable in our spectra.

In summary, we conclude that the abundance results reported here cannot be significantly affected by light from a third star in the system - certainly not the discrepant $\mathrm{Li}$ abundances of the two stars.

\section{Summary}

Our high-resolution VLT/UVES observations of the doublelined spectroscopic binary CS 22876-032 confirm that it harbours the most metal-poor dwarfs reported to date. Our improved orbital elements, together with published photometry and theoretical isochrones, enable us to determine stellar parameters of $T_{\text {eff, }}=6500 \pm 100 \mathrm{~K}$ and $\log g_{\mathrm{A}}=4.4 \pm 0.1$ for the primary (star A) and $T_{\text {eff }, \mathrm{B}}=5900 \pm 150 \mathrm{~K}$ and $\log g_{\mathrm{B}}=4.6 \pm 0.1$ for the secondary (star B).

Using 1D OSMARCS models and the TURBOSPECTRUM code, we determine abundances of $\mathrm{Li}, \mathrm{O}, \mathrm{Na}, \mathrm{Mg}, \mathrm{Al}, \mathrm{Si}, \mathrm{Ca}$, $\mathrm{Sc}, \mathrm{Ti}, \mathrm{Cr}, \mathrm{Mn}, \mathrm{Fe}, \mathrm{Co}$, and $\mathrm{Ni}$, correcting the observed spectra for the veiling from the continuum flux of the other star. We find $[\mathrm{Fe} / \mathrm{H}]=-3.66 \pm 0.16$ and $[\mathrm{Fe} / \mathrm{H}]=-3.57 \pm 0.25$ for star $\mathrm{A}$ and $\mathrm{B}$, respectively. Using $\mathrm{CO}^{5} \mathrm{BOLD}$ model atmospheres to estimate 3D abundance corrections, we compute full 3D spectrum synthesis using the Linfor3D code for $\mathrm{Li}$ and $\mathrm{O}$ to estimate the $3 \mathrm{D}-1 \mathrm{D}_{\mathrm{LHD}}$ corrections, while we use a horizontal and temporal average of the $3 \mathrm{D}$ model to compute $\langle 3 \mathrm{D}\rangle$ abundances with TURBOSPECTRUM for the rest of elements. In general, we find $\left(\langle 3 D\rangle-1 D_{\text {MARCS }}\right)$ corrections to be $\$ 0.1 \mathrm{dex}$, negative for neutral species and positive for ionised species; for $\mathrm{Fe}$ in particular, we find corrections to be $\sim-0.12$ (A) and $\sim-0.07$ (B).

The $[\alpha / \mathrm{Fe}]$ ratios are consistent with our earlier results for EMP giants (Cayrel et al. 2004, First Stars V), although Ca and $\mathrm{Si}$ are slightly low $([\mathrm{X} / \mathrm{Fe}] \lesssim 0)$, but actually consistent with our results for other EMP dwarfs (Bonifacio et al. 2007, in prep.). $[\mathrm{Na} / \mathrm{Fe}]$ appears consistent with both EMP giants and dwarfs when NLTE corrections are considered. The LTE value of $[\mathrm{Al} / \mathrm{Fe}]$ is not consistent with those in EMP giants, but $\mathrm{Al}$ is severely affected by NLTE effects, which may solve this discrepancy. The iron-peak elements follow the established trends in EMP giants and dwarfs.

Our high-quality spectra allowed us to measure the Li doublet in both stars of CS 22876-032 for the first time. We find NLTE Li abundances of $2.18 \pm 0.05$ and $1.77 \pm 0.09$ for stars $\mathrm{A}$ and $\mathrm{B}$, respectively. While the $\mathrm{Li}$ abundance of star A corresponds to the level of the Spite plateau, the secondary star has a significantly lower abundance. This discrepancy may be resolved by assuming that the secondary star has been subject to significant Li depletion, which, according to standard Li depletion isochrones, would have been the case if the star were $350 \mathrm{~K}$ cooler than assumed by our analysis. Full 3D corrections for $\mathrm{Li}$ are estimated to be $\sim-0.3$ (A) and $\sim-0.2(\mathrm{~B})$; however, these computations were performed in LTE, and 3D NLTE corrections are needed to confirm the sign and value of these corrections.

The near-UV part of our VLT/UVES spectra enabled us to measure oxygen abundances from the $\mathrm{OH}$ bands. We find $1 \mathrm{D}[\mathrm{O} / \mathrm{Fe}]$ values of $2.14 \pm 0.15(\mathrm{~A})$ and $1.81 \pm 0.33(\mathrm{~B})$ and compute full 3D corrections for the $\mathrm{OH}$ lines, which are -1.5 (A) and -1.0 dex (B). Using these corrections and the $\langle 3 \mathrm{D}\rangle \mathrm{Fe} \mathrm{I}$ abundances, we determine $3 \mathrm{D}[\mathrm{O} / \mathrm{Fe}]$ ratios of $0.77 \pm 0.15(\mathrm{~A})$ and $0.97 \pm 0.33(\mathrm{~B})$. These $3 \mathrm{D}[\mathrm{O} / \mathrm{Fe}]$ ratios are consistent with those derived from the [OI] line in EMP giants of similar metallicity, where $3 \mathrm{D}$ corrections should not be significant.
Acknowledgements. We are grateful to A. Chieffi and M. Limongi, for computing, at our request, isochrones appropriate for the metallicity of CS 22876-032 and for many interesting discussions on the evolution of extremely low metallicity dwarfs. J.I., P.B. and H.-G.L. acknowledge support from the EU contract MEXT-CT-2004-014265 (CIFIST). P.B. also acknowledges support from MIUR - PRIN grant 2004025729. B.N. and J.A. thank the Carlsberg Foundation and the Danish Natural Science Research Council for support for this work. T.C.B. and T.S. acknowledge partial support from the US National Science Foundation under grants AST 04-06784, AST 07-07776, as well as from grant PHY 02-15783; Physics Frontier Center/Joint Institute for Nuclear Astrophysics (JINA). B.N. and J.A. acknowledge support from the Carlsberg Foundation and the Danish Natural Science Research Council. This publication makes use of data products from the Two Micron All Sky Survey, which is a joint project of the University of Massachusetts and the Infrared Processing and Analysis Center/California Institute of Technology, funded by the National Aeronautics Ans Space Administration and the National Science Foundation. This work has also made use of the IRAF facilities and the SIMBAD database, operated at CDS, Strasbourg, France.

\section{References}

Alvarez, R., \& Plez, B. 1998, A\&A, 330, 1109

Andrievsky, S. M., Spite, M., \& Korotin, S. A. Å. 2007, A\&A, 464, 1081

Asplund, M., \& García Pérez, A. E. 2001, A\&A, 372, 601

Asplund, M., Gustafsson, B., Kiselman, D., \& Eriksson, K. 1997, A\&A, 318, 521

Asplund, M., Nordlund, Å., Trampedach, R., \& Stein, R. F. 1999, A\&A, 346, L17

Asplund, M., Carlsson, M., \& Botnen, A. V. 2003, A\&A, 399, L31

Asplund, M., Lambert, D. L., Nissen, P. E., Primas, F., \& Smith, V. V. 2006, ApJ, 644, 229

Baumüller, D., \& Gehren, T. 1997, A\&A, 325, 1088

Barklem, P. S., Piskunov, N., \& O'Mara, B. J. 2000, A\&A, 363, 1091

Barklem, P. S., Stempels, H. C., Allende Prieto, C., et al. 2002, A\&A, 385, 951

Beers, T. C., Preston, G. W., \& Shectman, S. A. 1985, AJ, 90, 2089

Bessell, M. S., \& Norris, J. 1984, ApJ, 285, 622

Bessell, M. S., \& Brett, J. M. 1988, PASP, 100, 1134

Bessell, M. S., Sutherland, R. S., \& Ruan, K. 1991, ApJ, 383, L71

Bessell, M. S., Castelli, F. \& Plez, B. 1998, A\&A, 333, 231

Boesgaard, A. M., \& Novicki, M. C. 2006, ApJ, 641, 1122

Boesgaard, A. M., Deliyannis, C. P., King, J. R., et al. 1999a, AJ, 117, 1549

Boesgaard, A. M., King, J. R., Deliyannis, C. P., \& Vogt, S. S. 1999b, AJ, 117, 492

Bonifacio, P., Castelli, F., \& Molaro, P. 1990, in proceedings of Chemical and Dynamical Evolution of Galaxies, ed. F. Ferrini, J. Franco, \& F. Matteucci (Pisa: ETS Editrice), 67

Bonifacio, P., \& Molaro, P. 1997, MNRAS, 285, 847

Bonifacio, P., Pasquini, L., Spite, F., et al. 2002, A\&A, 390, 91

Bonifacio, P., Molaro, P., \& Sivarani, T. A.. 2007, A\&A, 462, 851 (Paper VII)

Caffau, E., Faraggiana, R., Bonifacio, P., Ludwig, H.-G., \& Steffen, M. 2007a, A\&A, 470, 699

Caffau, E., Ludwig, H.-G., Steffen, M., et al. 2007b, A\&A, in preparation Carlsson, M., Rutten, R. J., Bruls, J. H. M. J., \& Shchukina, N. G. 1994, A\&A, 288,860

Carpenter, J. M. 2001, AJ, 121, 2851

Castelli, F., \& Kurucz, R. L. 2003, in IAU Symposium, ed. N. Piskunov, W. W. Weiss, \& D. F. Gray, 20

Castelli, F., \& Hubrig, S. 2004, A\&A, 425, 263

Cayrel, R. 1988, The Impact of Very High S/N Spectroscopy on Stellar Physics, IAU Symp., 132, 345

Cayrel, R., \& Steffen, M. 2000, The Light Elements and their Evolution, 198, 437

Cayrel, R., Depagne, E., \& Spite, M. Å. 2004, A\&A, 416, 1117 (Paper V)

Cayrel, R., Steffen, M., \& Chand, H. A.. 2007, A\&A, 473, L37

Charbonneau, P. 1995, ApJS, 101, 309

Charbonnel, C., \& Primas, F. 2005, A\&A, 442, 961

Cohen, J. G., Christlieb, N., \& McWilliam, A. Å. 2004, ApJ, 612, 1107

Collet, R., Asplund, M., \& Trampedach, R. 2007, A\&A, 469, 687

Deliyannis, C. P., Demarque, P., \& Kawaler, S. D. 1990, ApJS, 73, 21

Edvardsson, B., Andersen, J., Gustafsson, B., et al. 1993, A\&A, 275, 101

François, P., Depagne, E., \& Hill, V. Å. 2003, A\&A, 403, 1105 (Paper III)

François, P., Matteucci, F., Cayrel, R., et al. 2004, A\&A, 421, 613

Frebel, A., Aoki, W., Christlieb, N., et al. 2005, Nature, 434, 871

Freytag, B., Steffen, M., \& Dorch, B. 2002, Astron. Nachr., 323, 213

Fuhrmann, K., Axer, M., \& Gehren, T. 1993, A\&A, 271, 451

Gangrsky, Y., Marinova, K., Zemlyanoi, S., et al. 2006, Hyperfine Interactions, 171,209 
Gilmore, G., Gustafsson, B., Edvardsson, B., \& Nissen, P. E. 1992, Nature, 357, 379

Goldberg, D., Mazeh, T., \& Latham, D. W. A. 2002, AJ, 124, 1132

Grainge, K., Carreira, P., \& Cleary, K. Å. 2003, MNRAS, 341, L23

Grevesse, N., \& Sauval, A. 2000, Encyclopedia of Astronomy and Astrophysics, ed. P. Murdin, article 1979 (Bristol: Institute of Physics Publishing)

Gustafsson, B., Bell, R. A., Eriksson, K., \& Nordlund, Å. 1975, A\&A, 42, 407

Gustafsson, B., Edvardsson, B., Eriksson, K., et al. 2003, in Stellar Atmosphere Modeling, ed. I. Hubeny, D. Mihalas, \& K. Werner, ASP Conf. Ser., 288, 331

Harrington, R. S. 1977, Rev. Mex. Astron. Astrofis., 3, 139

Israelian, G., García López, R. J., \& Rebolo, R. 1998, ApJ, 507, 805

Israelian, G., Rebolo, R., García López, R. J., et al. 2001, ApJ, 551, 833

Khodykin, S. A., Zakharov, A. I., \& Andersen, W. L. 2004, ApJ, 615, 506

Korn, A. J., Grundahl, F., Richard, O., et al. 2006, Nature, 442, 657

Korn, A. J., Grundahl, F., Richard, O., et al. 2007, ApJ, in press [arXiv:0709.0639]

Kurucz, R. 1993a, ATLAS9 Stellar Atmosphere Programs and $2 \mathrm{~km} \mathrm{~s}^{-1}$ grid. Kurucz CD-ROM No. 13, Cambridge, Mass.: Smithsonian Astrophysical Observatory

Kurucz, R. L. 2005a, Mem. Soc. Astron. Ital. Suppl., 8, 14

Kuo, C. L., Ade, P. A. R., \& Bock, J. J. A.. 2004, ApJ, 600, 32

Latham, D. W., Stefanik, R. P., \& Torres, G. Å. 2002, AJ, 124, 1144

Lomb, N. R. 1976, Ap\&SS, 39, 447

Ludwig, H.-G., \& Steffen, M. 2007, to appear in the Proceedings of the ESO/Lisbon/Aveiro Workshop Precision Spectroscopy in Astrophysics, ed. L. Pasquini, M. Romaniello, N. C. Santos, \& A. Correia [arXiv: 0704 . 1176] McWilliam, A., Preston, G. W., Sneden, C., \& Searle, L. 1995, AJ, 109, 2757

Meléndez, J., \& Ramírez, I. 2004, ApJ, 615, L33

Molaro, P., \& Castelli, F. 1990, A\&A, 228, 426

Molaro, P., Bonifacio, P., Castelli, F., \& Pasquini, L. 1997, A\&A, 319, 593

Nissen, P. E. 1989, The Messenger, 58, 40
Nissen, P. E., Primas, F., Asplund, M., \& Lambert, D. L. 2002, A\&A, 390, 235 Norris, J. E., Peterson, R. C., \& Beers, T. C. 1993, ApJ, 415, 797

Norris, J. E., Beers, T. C., \& Ryan, S. G. 2000, ApJ, 540, 456

Pearson, T. J., Mason, B. S., \& Readhead, A. C. S. A. 2003, ApJ, 591, 556 Pickering, J. C. 1996, ApJS, 107, 811

Plez, B., Brett, J. M., \& Nordlund, A. 1992, A\&A, 256, 551

Preston, G. W., Shectman, S. A., \& Beers, T. C. 1991, ApJS, 76, 1001 Primas, F. 2002, Ap\&SS, 281, 195

Richard, O., Michaud, G., Richer, J., et al. 2002, ApJ, 568, 979

Richard, O., Michaud, G., \& Richer, J. 2005, ApJ, 619, 538

Rebolo, R., Battye, R. A., Carreira, P., et al. 2004, MNRAS, 353, 747

Ryan, S. G., Norris, J. E., Bessell, M. S., \& Deliyannis, C. 1992, ApJ, 388, 184 Ryan, S. G., Norris, J. E., \& Beers, T. C. 1999, ApJ, 523, 654

Sbordone, L. 2005, Mem. Soc. Astron. Ital. Suppl., 8, 61

Sbordone, L., Bonifacio, P., Castelli, F., \& Kurucz, R. L. 2004, Mem. Soc. Astron. Ital. Suppl., 5, 93

Scargle, J. D. 1982, ApJ, 263, 835

Schuster, W. J., Parrao, L., \& Contreras Martinez, M. E. 1993, A\&AS, 97, 951 Schuster, W. J., Nissen, P. E., Parrao, L., Beers, T. C., \& Overgaard, L. P. 1996, A\&AS, 117, 317

Shchukina, N. G., Trujillo Bueno, J., \& Asplund, M. 2005, ApJ, 618, 939

Slettebak, A., \& Brundage, R. K. 1971, AJ, 76, 338

Spergel, D. N., Bean, R., \& Doré, O. Å. 2003, ApJS, 148, 175

Spergel, D. N., Verde, L., \& Peiris, H. V. Å. 2007, ApJS, 170, 377

Spite, F., \& Spite, M. 1982a, A\&A, 115, 357

Spite, M., \& Spite, F. 1982b, Nature, 297, 483

van't Veer-Menneret, C., \& Mégessier, C. 1996, A\&A, 309, 879

Wahlgren, G. M. 2005, Mem. Soc. Astron. Ital. Suppl., 8, 108

Wedemeyer, S., Freytag, B., Steffen, M., Ludwig, H.-G., \& Holweger, H. 2003, Astron. Nachr., 324, 410

Wichmann, R., Schmitt, J. H. M. M., \& Hubrig, S. 2003, A\&A, 400, 293 
J. I. González Hernández et al.: Chemical abundances of EMP dwarfs in CS 22876-032, Online Material $p 1$

Table 1. Radial-velocity observations of CS 22876-032. For each velocity, $\sigma$ is the estimated error and (O-C) the residual from the orbital fit. ${ }^{\star}: \mathrm{H}_{\alpha}$ velocities; omitted from solution.

\begin{tabular}{|c|c|c|c|c|c|c|c|c|c|}
\hline $\begin{array}{l}\text { Date } \\
\text { (days) }\end{array}$ & HJD-2 400000 & $\begin{array}{l}\text { Phase } \\
\mathrm{km} \mathrm{s}^{-1}\end{array}$ & $\begin{array}{r}V_{\mathrm{A}} \\
\mathrm{km} \mathrm{s}^{-1}\end{array}$ & $\begin{array}{r}\sigma V_{\mathrm{A}} \\
\mathrm{km} \mathrm{s}^{-1}\end{array}$ & $\begin{array}{r}(\mathrm{O}-\mathrm{C})_{\mathrm{A}} \\
\mathrm{km} \mathrm{s}^{-1}\end{array}$ & $\begin{array}{r}V_{\mathrm{B}} \\
\mathrm{km} \mathrm{s}^{-1}\end{array}$ & $\begin{array}{r}\sigma V_{\mathrm{B}} \\
\mathrm{km} \mathrm{s}^{-1}\end{array}$ & $(\mathrm{O}-\mathrm{C})_{\mathrm{B}}$ & Source \\
\hline 1985 Sep. $6 \ldots \ldots$. & 46315.1 & 0.669 & -83.9 & 2.0 & -3.44 & - & - & - & NBR00 \\
\hline 1985 Dec. $16 \ldots \ldots$ & 46416.0 & 0.907 & -96.8 & 2.0 & 1.72 & - & - & - & NBR00 \\
\hline 1988 Sep. 27 ...... & 47431.7 & 0.297 & -93.5 & 1.0 & 1.44 & - & - & - & MC90 \\
\hline 1989 Sep. 13 ...... & 47783.2 & 0.124 & -107.8 & 1.0 & 0.63 & -74.8 & 1.0 & 1.48 & NBR00 \\
\hline 1989 Oct. $15 \ldots \ldots$ & 47814.6 & 0.199 & -104.3 & 1.0 & -0.94 & - & - & - & NBR00 \\
\hline 1989 Oct. $16 \ldots \ldots$ & 47815.7 & 0.201 & -103.1 & 1.0 & 0.07 & -81.2 & 1.0 & 0.86 & NBR00 \\
\hline 1989 Oct. $17 \ldots \ldots$ & 47816.6 & 0.204 & -102.1 & 1.0 & 0.87 & - & - & - & NBR00 \\
\hline 1989 Dec. 6 ........ & 47867.0 & 0.322 & -93.3 & 1.0 & -0.35 & - & - & - & NBR00 \\
\hline 1990 Sep. 1 ....... & 48135.7 & 0.955 & -103.0 & 1.0 & 0.83 & - & - & - & CASPEC \\
\hline 1990 Sep. 27 ...... & 48162.1 & 0.017 & -109.6 & 1.0 & -0.91 & -76.2 & 1.0 & -0.20 & NBR00 \\
\hline 1991 May 23 ....... & $48400.0^{\star}$ & 0.577 & -87.5 & 2.0 & -7.34 & - & - & - & EMMI \\
\hline 1991 Aug. 22 ...... & 48490.5 & 0.790 & -87.0 & 2.0 & -0.47 & - & - & - & NBR00 \\
\hline 1991 Sep. 21 ...... & 48520.7 & 0.861 & -94.0 & 1.0 & -0.65 & - & - & - & EMMI \\
\hline 1992 Aug. 17 ...... & 48851.8 & 0.640 & -78.6 & 1.0 & 1.41 & -107.6 & 1.0 & -0.12 & NBR00 \\
\hline 1992 Dec. $12 \ldots \ldots$ & $48967.5^{\star}$ & 0.912 & -90.9 & 2.0 & 8.33 & - & - & - & EMMI \\
\hline 1996 Aug. 7 ....... & 50303.1 & 0.057 & -110.2 & 1.0 & -0.28 & -76.2 & 1.0 & -1.55 & NBR00 \\
\hline 1997 Aug. 23 ...... & 50683.8 & 0.957 & -104.2 & 1.0 & -0.57 & -81.3 & 1.0 & 0.25 & NBR00 \\
\hline 1998 Aug. 12 ...... & 51038.3 & 0.787 & -87.8 & 1.0 & -1.47 & -101.1 & 1.0 & -0.56 & NBR00 \\
\hline 1999 Jul. 29 ...... & 51388.7 & 0.612 & -77.0 & 2.0 & 2.89 & _- & - & - & NBR00 \\
\hline 1999 Sep. 23 ...... & 51444.6 & 0.744 & -84.3 & 1.0 & -0.93 & -105.5 & 1.0 & -1.71 & NBR00 \\
\hline 2000 Jul. $19 \ldots \ldots$ & 51744.8 & 0.451 & -84.9 & 0.5 & -0.52 & -102.9 & 0.3 & -0.22 & VLT \\
\hline 2000 Jul. 20 ....... & 51745.8 & 0.453 & -83.7 & 0.4 & 0.56 & -102.3 & 0.8 & 0.51 & VLT \\
\hline 2000 Aug. 3 ........ & 51759.8 & 0.486 & -82.6 & 0.3 & 0.13 & -103.9 & 0.5 & 0.59 & VLT \\
\hline 2000 Aug. $11 \ldots \ldots$ & 51767.9 & 0.505 & -82.3 & 0.3 & -0.31 & -105.3 & 0.3 & 0.01 & VLT \\
\hline 2000 Oct. 17 ....... & 51834.7 & 0.662 & -80.1 & 0.3 & 0.22 & -107.0 & 0.4 & 0.14 & VLT \\
\hline 2000 Oct. $20 \ldots \ldots$ & 51837.6 & 0.669 & -79.9 & 1.0 & 0.56 & -105.6 & 1.4 & 1.38 & VLT \\
\hline 2001 Nov. 7 ........ & 52220.6 & 0.571 & -80.6 & 0.3 & -0.35 & -107.4 & 0.3 & -0.18 & VLT \\
\hline 2001 Nov. 8 ........ & 52221.6 & 0.573 & -80.2 & 0.3 & 0.01 & -107.2 & 0.3 & 0.06 & VLT \\
\hline 2001 Nov. 9 ........ & 52222.6 & 0.575 & -79.4 & 0.7 & 0.78 & -106.6 & 0.9 & 0.69 & VLT \\
\hline
\end{tabular}

Sources: NBR00: Norris et al. (2000, and references therein); MC90: Molaro \& Castelli (1990); CASPEC: unpublished velocity from a Caspec spetrum; EMMI: unpublished velocities from EMMI spectra; VLT: this paper. 


\section{Appendix A: Veiling factors for a triple system}

We derive here the expression for the veiling factors of a triple system which are a trivial extension of those for a double system, however since they are not readily found in any paper or book we know of, we provide them here for the reader's convienience.

We shall use the following notation:

\begin{tabular}{|c|c|}
\hline & \\
\hline 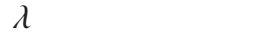 & wavelength \\
\hline$s_{i}(i=1,2,3)$ & $\begin{array}{l}\text { the flux spectrum } s_{\lambda} \text { of } \\
\text { the } i \text { th component }\end{array}$ \\
\hline$c_{i}(i=1,2,3)$ & $\begin{array}{l}\text { the continuum flux spectrum } c_{\lambda} \text { of } \\
\text { the } i \text { th component }\end{array}$ \\
\hline$d_{i}(i=1,2,4)$ & the line depression $=c_{i}-s_{i}$ \\
\hline$E W_{i}(i=1,2,3)$ & $\begin{array}{l}\text { the intrinsic equivalent width of a } \\
\text { spectral line of the } i \text { th component }\end{array}$ \\
\hline$E W_{i}^{\mathrm{obs}}(i=1,2,3)$ & $\begin{array}{l}\text { the observed equivalent width of a } \\
\text { spectral line of the } i \text { th component }\end{array}$ \\
\hline$E W^{\text {obs }}$ & $\begin{array}{l}\text { the observed equivalent width of the } \\
\text { three components. }\end{array}$ \\
\hline
\end{tabular}

In any orbital phase we have:

$E W_{123}^{\mathrm{obs}}=\int_{\lambda 1}^{\lambda 2} \frac{d_{1}+d_{2}+d_{3}}{c_{1}+c_{2}+c_{3}} \mathrm{~d} \lambda$.

Where the interval $[\lambda 1, \lambda 2]$ includes the desired spectral feauture, which we assume to be isolated. Considering that the continuum fluxes can be assumed to be constant over the integration interval this can be re-written as:

$$
\begin{aligned}
E W_{123}^{\mathrm{obs}}= & \frac{c_{1}}{c_{1}+c_{2}+c_{3}} \int_{\lambda 1}^{\lambda 2} \frac{d_{1}}{c_{1}} \mathrm{~d} \lambda+\frac{c_{2}}{c_{1}+c_{2}+c_{3}} \int_{\lambda 1}^{\lambda 2} \frac{d_{2}}{c_{2}} \mathrm{~d} \lambda \\
& +\frac{c_{3}}{c_{1}+c_{2}+c_{3}} \int_{\lambda 1}^{\lambda 2} \frac{d_{3}}{c_{3}} \mathrm{~d} \lambda .
\end{aligned}
$$

We now define the veiling factors as $f_{i}=\left(c_{1}+c_{2}+c_{3}\right) / c_{i}$ and consider a phase in which the radial velocities are such that the line of of each component is isolated and not blended with the others, then one has:

$E W_{123}^{\mathrm{obs}}=E W_{1}^{\mathrm{obs}}+E W_{2}^{\mathrm{obs}}+E W_{3}^{\mathrm{obs}}$

and noting that:

$\int_{\lambda 1}^{\lambda 2} \frac{d_{i}}{c_{i}} \mathrm{~d} \lambda=E W_{i}$

one has that:

$E W_{1}^{\mathrm{obs}}+E W_{2}^{\mathrm{obs}}+E W_{3}^{\mathrm{obs}}=E W_{1} / f_{1}+E W_{2} / f_{2}+E W_{3} / f_{3}$

finally considering that in this phase the lines are not overlapping this equality implies:

$E W_{i}=f_{i} E W_{i}^{\mathrm{obs}}$. 
J. I. González Hernández et al.: Chemical abundances of EMP dwarfs in CS 22876-032, Online Material p 3

Table A.1. Line data, equivalent widths, veiling factors and 1D abundances of CS 22876-032A, B.

\begin{tabular}{|c|c|c|c|c|c|c|c|c|c|}
\hline Specie & $\begin{array}{l}\lambda \\
(\mathrm{nm})\end{array}$ & $\begin{array}{l}\chi \\
(\mathrm{eV})\end{array}$ & $\log g f$ & $\begin{array}{r}E W_{\mathrm{A}, \mathrm{obs}} \\
(\mathrm{pm})\end{array}$ & $f_{\mathrm{A}, 1 \mathrm{D}}$ & $\begin{array}{r}A(\mathrm{X})_{\mathrm{A}, 1 \mathrm{D}} \\
(\mathrm{dex})\end{array}$ & $\begin{array}{r}E W_{\mathrm{B}, \mathrm{obs}} \\
(\mathrm{pm})\end{array}$ & $f_{\mathrm{B}, 1 \mathrm{D}}$ & $\begin{array}{r}A(\mathrm{X})_{\mathrm{B}, 1 \mathrm{D}} \\
(\mathrm{dex})\end{array}$ \\
\hline $\mathrm{NaI}$ & 588.9951 & 0.000 & 0.112 & 1.40 & 1.35 & 2.63 & - & - & - \\
\hline $\mathrm{NaI}$ & 589.5924 & 0.000 & -0.191 & 0.79 & 1.35 & 2.64 & 0.49 & 3.87 & 2.54 \\
\hline $\operatorname{Mg}_{I}$ & 333.6674 & 2.720 & -1.230 & 1.07 & 1.32 & 4.57 & 0.90 & 4.16 & 4.68 \\
\hline Mg I & 382.9355 & 2.710 & -0.207 & 5.01 & 1.31 & 4.41 & 2.06 & 4.24 & 4.29 \\
\hline Mg I & 383.2304 & 2.710 & 0.146 & 6.03 & 1.31 & 4.29 & 2.87 & 4.25 & 4.35 \\
\hline $\mathrm{Mg}_{\mathrm{I}}$ & 383.8290 & 2.720 & 0.415 & 6.89 & 1.30 & 4.21 & 2.77 & 4.31 & 4.06 \\
\hline $\mathrm{Mg}_{\mathrm{I}}$ & 416.7271 & 4.340 & -1.000 & 0.23 & 1.29 & 4.84 & 0.11 & 4.41 & 4.86 \\
\hline Mg I & 517.2684 & 2.710 & -0.380 & 4.88 & 1.33 & 4.47 & 2.42 & 4.04 & 4.41 \\
\hline Mg I & 518.3604 & 2.720 & -0.158 & 5.73 & 1.33 & 4.43 & 3.24 & 4.03 & 4.52 \\
\hline Mg I & 552.8405 & 4.340 & -0.341 & 0.60 & 1.34 & 4.58 & 0.16 & 3.94 & 4.25 \\
\hline $\mathrm{Mg}_{\mathrm{I}}$ & 880.6756 & 4.340 & -0.137 & 0.81 & 1.39 & 4.41 & 0.62 & 3.57 & 4.50 \\
\hline $\mathrm{Al}$ I & 394.4006 & 0.000 & -0.623 & 1.51 & 1.28 & 2.74 & 1.12 & 4.54 & 2.88 \\
\hline $\mathrm{Al}$ I & 396.1520 & 0.010 & -0.323 & 1.41 & 1.31 & 2.42 & 1.16 & 4.24 & 2.56 \\
\hline Si I & 390.5523 & 1.910 & -1.090 & 1.55 & 1.29 & 3.80 & 1.32 & 4.42 & 4.07 \\
\hline $\mathrm{CaI}$ & 422.6728 & 0.000 & 0.240 & 3.60 & 1.30 & 2.71 & 1.70 & 4.39 & 2.68 \\
\hline Ca II & 317.9331 & 3.150 & 0.512 & 4.08 & 1.30 & 2.62 & 1.36 & 4.28 & 2.57 \\
\hline Ca II & 370.6024 & 3.120 & -0.480 & 1.41 & 1.34 & 2.58 & - & - & - \\
\hline Ca II & 373.6902 & 3.150 & -0.173 & 3.22 & 1.33 & 2.84 & 0.68 & 4.04 & 2.53 \\
\hline Sc II & 361.3829 & 0.020 & 0.416 & 0.42 & 1.33 & -0.69 & 0.42 & 4.01 & -0.40 \\
\hline Sc II & 424.6822 & 0.310 & 0.240 & 0.37 & 1.30 & -0.44 & 0.16 & 4.38 & -0.41 \\
\hline Ti II & 316.8532 & 0.150 & -0.310 & 2.35 & 1.30 & 1.66 & 0.70 & 4.29 & 1.34 \\
\hline Ti II & 323.4520 & 0.050 & 0.426 & 4.55 & 1.31 & 1.54 & 1.64 & 4.25 & 1.34 \\
\hline Ti II & 323.6578 & 0.030 & 0.234 & 4.11 & 1.31 & 1.56 & 1.72 & 4.25 & 1.59 \\
\hline Ti II & 324.1994 & 0.000 & -0.045 & 3.20 & 1.31 & 1.51 & 1.75 & 4.24 & 1.86 \\
\hline Ti II & 325.1918 & 0.010 & -0.579 & 1.54 & 1.31 & 1.52 & 0.65 & 4.23 & 1.39 \\
\hline Ti II & 332.2941 & 0.150 & -0.093 & 2.38 & 1.32 & 1.44 & 1.23 & 4.17 & 1.53 \\
\hline Ti II & 332.9453 & 0.140 & -0.274 & 2.53 & 1.32 & 1.65 & 1.33 & 4.17 & 1.78 \\
\hline Ti II & 338.0279 & 0.050 & -0.570 & 3.38 & 1.32 & 2.12 & 1.33 & 4.14 & 1.98 \\
\hline Ti II & 338.7846 & 0.030 & -0.432 & 2.29 & 1.32 & 1.63 & 1.01 & 4.14 & 1.54 \\
\hline Ti II & 344.4314 & 0.150 & -0.810 & 1.02 & 1.32 & 1.63 & - & - & - \\
\hline Ti II & 345.6388 & 2.060 & -0.230 & 0.41 & 1.32 & 2.32 & - & - & - \\
\hline Ti II & 347.7187 & 0.120 & -0.967 & 1.17 & 1.32 & 1.83 & 0.58 & 4.08 & 1.76 \\
\hline Ti II & 348.9741 & 0.140 & -1.920 & 0.40 & 1.33 & 2.27 & - & - & - \\
\hline Ti II & 350.0340 & 0.120 & -2.020 & 0.37 & 1.33 & 2.32 & - & - & - \\
\hline Ti II & 375.9296 & 0.610 & 0.270 & 2.39 & 1.32 & 1.33 & 0.94 & 4.16 & 1.26 \\
\hline Ti II & 376.1323 & 0.570 & 0.170 & 2.36 & 1.32 & 1.39 & 1.07 & 4.16 & 1.43 \\
\hline Ti II & 391.3468 & 1.120 & -0.410 & 0.39 & 1.28 & 1.48 & - & - & - \\
\hline Ti II & 402.8343 & 1.890 & -0.990 & 0.15 & 1.29 & 2.30 & - & - & - \\
\hline Ti II & 518.8680 & 1.580 & -1.050 & 0.14 & 1.33 & 2.01 & - & - & - \\
\hline $\mathrm{CrI}$ & 357.8684 & 0.000 & 0.409 & 0.85 & 1.33 & 1.79 & 0.61 & 4.03 & 1.61 \\
\hline $\mathrm{CrI}$ & 425.4332 & 0.000 & -0.110 & 0.45 & 1.30 & 1.83 & 0.28 & 4.37 & 1.63 \\
\hline CrI & 427.4796 & 0.000 & -0.230 & 0.32 & 1.30 & 1.79 & 0.16 & 4.36 & 1.48 \\
\hline CrI & 428.9716 & 0.000 & -0.360 & 0.46 & 1.30 & 2.09 & 0.58 & 4.35 & 2.27 \\
\hline CrI & 520.6038 & 0.940 & 0.020 & 0.13 & 1.33 & 1.92 & - & - & - \\
\hline Cr II & 313.2053 & 2.480 & 0.451 & 2.85 & 1.30 & 2.29 & 1.10 & 4.32 & 2.37 \\
\hline Mn II & 344.1988 & 1.780 & -0.270 & 0.54 & 1.32 & 1.38 & 0.32 & 4.11 & 1.53 \\
\hline Fe I & 347.5450 & 0.090 & -1.054 & 3.24 & 1.32 & 3.99 & 1.89 & 4.09 & 4.07 \\
\hline Fe I & 347.6702 & 0.120 & -1.507 & 1.96 & 1.32 & 4.05 & 1.40 & 4.08 & 4.07 \\
\hline Fe I & 349.0574 & 0.050 & -1.105 & 2.91 & 1.33 & 3.90 & 1.93 & 4.07 & 4.11 \\
\hline Fe I & 356.5379 & 0.960 & -0.133 & 2.96 & 1.33 & 3.75 & 1.90 & 4.04 & 3.92 \\
\hline $\mathrm{Fe} I$ & 358.1193 & 0.860 & 0.406 & 4.17 & 1.33 & 3.55 & 2.81 & 4.03 & 3.89 \\
\hline $\mathrm{Fe} I$ & 360.8859 & 1.010 & -0.100 & 3.39 & 1.33 & 3.91 & 2.17 & 4.01 & 4.13 \\
\hline Fe I & 361.8768 & 0.990 & -0.003 & 3.55 & 1.33 & 3.85 & 2.25 & 4.00 & 4.06 \\
\hline $\mathrm{Fe} \mathrm{I}$ & 364.7843 & 0.920 & -0.194 & 3.29 & 1.33 & 3.72 & 2.24 & 4.00 & 4.09 \\
\hline
\end{tabular}


Table A.1. continued.

\begin{tabular}{|c|c|c|c|c|c|c|c|c|c|}
\hline Specie & $\begin{array}{l}\lambda \\
(\mathrm{nm})\end{array}$ & $\begin{array}{l}x \\
(\mathrm{eV})\end{array}$ & $\log g f$ & $\begin{array}{r}E W_{\mathrm{A}, \mathrm{obs}} \\
(\mathrm{pm})\end{array}$ & $f_{\mathrm{A}, 1 \mathrm{D}}$ & $\begin{array}{r}\mathrm{A}(\mathrm{X})_{\mathrm{A}, 1 \mathrm{D}} \\
(\mathrm{dex})\end{array}$ & $\begin{array}{r}E W_{\text {B,obs }} \\
\text { (pm) }\end{array}$ & $f_{\mathrm{B}, 1 \mathrm{D}}$ & $\begin{array}{r}\mathrm{A}(\mathrm{X})_{\mathrm{B}, 1 \mathrm{D}} \\
(\mathrm{dex})\end{array}$ \\
\hline $\mathrm{Fe} \mathrm{I}$ & 381.5840 & 1.490 & 0.237 & 3.79 & 1.30 & 3.88 & 1.61 & 4.34 & 3.77 \\
\hline $\mathrm{Fe} I$ & 382.0425 & 0.860 & 0.119 & 5.15 & 1.30 & 3.89 & 2.00 & 4.30 & 3.63 \\
\hline $\mathrm{Fe} I$ & 382.5881 & 0.920 & -0.037 & 4.10 & 1.31 & 3.76 & 2.00 & 4.26 & 3.82 \\
\hline $\mathrm{Fe} I$ & 382.7823 & 1.560 & 0.062 & 2.57 & 1.31 & 3.79 & 1.29 & 4.25 & 3.71 \\
\hline $\mathrm{Fe} I$ & 384.0438 & 0.990 & -0.506 & 2.26 & 1.30 & 3.76 & 1.11 & 4.34 & 3.60 \\
\hline $\mathrm{Fe} I$ & 384.1048 & 1.610 & -0.045 & 2.05 & 1.30 & 3.78 & 0.88 & 4.35 & 3.54 \\
\hline $\mathrm{Fe} I$ & 384.9967 & 1.010 & -0.871 & 1.26 & 1.29 & 3.79 & 1.09 & 4.46 & 3.99 \\
\hline $\mathrm{Fe} I$ & 385.6372 & 0.050 & -1.286 & 3.23 & 1.28 & 3.96 & 1.76 & 4.52 & 4.16 \\
\hline $\mathrm{Fe} I$ & 385.9911 & 0.000 & -0.710 & 5.44 & 1.28 & 4.03 & 2.42 & 4.54 & 4.08 \\
\hline $\mathrm{Fe} I$ & 386.5523 & 1.010 & -0.982 & 1.03 & 1.28 & 3.79 & - & - & - \\
\hline $\mathrm{Fe} I$ & 387.2501 & 0.990 & -0.928 & 1.58 & 1.29 & 3.95 & 0.99 & 4.49 & 3.94 \\
\hline $\mathrm{Fe} I$ & 387.8018 & 0.960 & -0.914 & 1.59 & 1.30 & 3.91 & 1.29 & 4.36 & 4.13 \\
\hline $\mathrm{Fe} I$ & 388.6282 & 0.050 & -1.076 & 2.64 & 1.32 & 3.60 & 2.18 & 4.17 & 4.17 \\
\hline $\mathrm{Fe} I$ & 388.7048 & 0.920 & -1.144 & 1.02 & 1.32 & 3.87 & 1.15 & 4.16 & 4.15 \\
\hline $\mathrm{Fe} I$ & 389.5656 & 0.110 & -1.670 & 1.59 & 1.31 & 3.92 & 1.43 & 4.19 & 4.15 \\
\hline $\mathrm{Fe} I$ & 389.9707 & 0.090 & -1.531 & 2.16 & 1.31 & 3.94 & 1.66 & 4.28 & 4.23 \\
\hline $\mathrm{Fe} I$ & 390.2946 & 1.560 & -0.466 & 1.10 & 1.30 & 3.79 & 0.91 & 4.35 & 3.93 \\
\hline $\mathrm{Fe} I$ & 390.6480 & 0.110 & -2.243 & 0.77 & 1.29 & 4.09 & 0.63 & 4.44 & 4.05 \\
\hline $\mathrm{Fe} I$ & 392.0258 & 0.120 & -1.746 & 1.39 & 1.28 & 3.91 & 1.28 & 4.58 & 4.20 \\
\hline Fe I & 392.7920 & 0.110 & -1.522 & 2.26 & 1.28 & 3.97 & 1.44 & 4.58 & 4.12 \\
\hline $\mathrm{Fe} I$ & 404.5812 & 1.490 & 0.280 & 3.96 & 1.29 & 3.85 & 1.38 & 4.47 & 3.52 \\
\hline $\mathrm{Fe} I$ & 406.3594 & 1.560 & 0.062 & 2.88 & 1.29 & 3.84 & 1.49 & 4.47 & 3.90 \\
\hline $\mathrm{Fe} I$ & 407.1738 & 1.610 & -0.022 & 2.54 & 1.29 & 3.87 & 1.26 & 4.44 & 3.83 \\
\hline $\mathrm{Fe} I$ & 413.2058 & 1.610 & -0.675 & 0.98 & 1.29 & 3.96 & 0.67 & 4.42 & 3.96 \\
\hline $\mathrm{Fe} I$ & 414.3868 & 1.560 & -0.511 & 1.24 & 1.29 & 3.87 & 1.07 & 4.43 & 4.09 \\
\hline $\mathrm{Fe} I$ & 420.2029 & 1.490 & -0.708 & 0.93 & 1.29 & 3.85 & 0.70 & 4.40 & 3.89 \\
\hline $\mathrm{Fe} I$ & 425.0787 & 1.560 & -0.714 & 0.58 & 1.30 & 3.69 & 0.49 & 4.37 & 3.74 \\
\hline $\mathrm{Fe} I$ & 426.0474 & 2.400 & 0.109 & 0.72 & 1.30 & 3.71 & 0.28 & 4.37 & 3.43 \\
\hline $\mathrm{Fe} I$ & 427.1761 & 1.490 & -0.164 & 2.43 & 1.30 & 3.86 & 1.23 & 4.36 & 3.78 \\
\hline $\mathrm{Fe} I$ & 432.5762 & 1.610 & 0.006 & 2.19 & 1.31 & 3.73 & 1.39 & 4.19 & 3.81 \\
\hline Fe II & 318.6738 & 1.700 & -1.710 & 1.69 & 1.30 & 4.13 & 0.90 & 4.28 & 4.37 \\
\hline Fe II & 319.2909 & 1.670 & -1.950 & 1.37 & 1.31 & 4.20 & 0.29 & 4.27 & 3.87 \\
\hline Fe II & 319.3799 & 1.720 & -1.720 & 1.30 & 1.31 & 4.01 & 0.70 & 4.27 & 4.21 \\
\hline Fe II & 319.6070 & 1.670 & -1.660 & 1.61 & 1.31 & 4.03 & 0.44 & 4.27 & 3.80 \\
\hline Fe II & 321.0444 & 1.720 & -1.790 & 2.05 & 1.31 & 4.36 & 0.95 & 4.26 & 4.52 \\
\hline Fe II & 321.3309 & 1.700 & -1.230 & 1.62 & 1.31 & 3.62 & - & - & - \\
\hline Fe II & 322.7742 & 1.670 & -1.130 & 3.29 & 1.31 & 4.05 & 1.94 & 4.26 & 4.81 \\
\hline $\mathrm{Fe}$ II & 325.5887 & 0.990 & -2.500 & 1.47 & 1.31 & 4.19 & 0.76 & 4.22 & 4.31 \\
\hline Fe II & 327.7348 & 0.990 & -2.470 & 1.33 & 1.31 & 4.11 & 0.72 & 4.20 & 4.23 \\
\hline Fe II & 423.3172 & 2.580 & -1.900 & 0.51 & 1.30 & 4.26 & - & - & - \\
\hline Fe II & 492.3927 & 2.890 & -1.320 & 0.36 & 1.32 & 3.77 & - & - & - \\
\hline $\mathrm{Fe}$ II & 501.8440 & 2.890 & -1.220 & 0.42 & 1.33 & 3.73 & 0.27 & 4.08 & 4.05 \\
\hline Fe II & 516.9033 & 2.890 & -0.870 & 0.65 & 1.33 & 3.58 & - & - & - \\
\hline CoI & 340.5114 & 0.430 & 0.250 & 1.17 & 1.32 & 1.93 & 2.44 & 4.12 & 2.10 \\
\hline Co I & 341.2333 & 0.510 & 0.030 & 1.09 & 1.32 & 2.05 & 0.86 & 4.12 & 2.02 \\
\hline CoI & 345.3508 & 0.430 & 0.380 & 1.54 & 1.32 & 1.99 & 1.41 & 4.11 & 1.95 \\
\hline CoI & 349.5681 & 0.630 & -0.270 & 0.65 & 1.33 & 2.11 & - & - & - \\
\hline Co I & 350.2278 & 0.430 & 0.070 & 1.40 & 1.33 & 2.11 & - & - & - \\
\hline CoI & 399.5302 & 0.920 & -0.220 & 0.24 & 1.29 & 2.00 & 0.19 & 4.48 & 1.94 \\
\hline Co I & 412.1311 & 0.920 & -0.320 & 0.18 & 1.31 & 1.90 & - & - & - \\
\hline $\mathrm{Ni}$ & 339.2983 & 0.030 & -0.540 & 2.66 & 1.32 & 2.90 & 1.57 & 4.13 & 2.92 \\
\hline $\mathrm{Ni} I$ & 343.3554 & 0.030 & -0.668 & 1.89 & 1.32 & 2.77 & 1.48 & 4.10 & 2.93 \\
\hline $\mathrm{Ni} I$ & 345.2885 & 0.110 & -0.910 & 1.14 & 1.32 & 2.78 & 1.13 & 4.11 & 2.93 \\
\hline $\mathrm{Ni} I$ & 345.8456 & 0.210 & -0.223 & 2.25 & 1.32 & 2.62 & 1.32 & 4.10 & 2.51 \\
\hline $\mathrm{Ni} I$ & 346.1649 & 0.030 & -0.347 & 2.55 & 1.32 & 2.67 & 1.51 & 4.10 & 2.66 \\
\hline $\mathrm{Ni} I$ & 349.2954 & 0.110 & -0.250 & 2.39 & 1.33 & 2.59 & 1.76 & 4.07 & 2.84 \\
\hline $\mathrm{Ni} I$ & 351.5049 & 0.110 & -0.211 & 2.74 & 1.33 & 2.67 & 1.74 & 4.06 & 2.77 \\
\hline $\mathrm{Ni}$ I & 352.4535 & 0.030 & 0.008 & 3.90 & 1.33 & 2.77 & 2.12 & 4.05 & 2.80 \\
\hline $\mathrm{Ni} I$ & 361.0461 & 0.110 & -1.149 & 0.95 & 1.33 & 2.92 & 1.07 & 4.01 & 3.05 \\
\hline $\mathrm{Ni} I$ & 361.9386 & 0.420 & 0.035 & 2.02 & 1.33 & 2.46 & 1.72 & 4.00 & 2.75 \\
\hline $\mathrm{Ni} I$ & 380.7138 & 0.420 & -1.180 & 1.11 & 1.30 & 3.17 & 0.30 & 4.38 & 2.58 \\
\hline $\mathrm{Ni} I$ & 385.8292 & 0.420 & -0.970 & 0.73 & 1.28 & 2.74 & 0.68 & 4.54 & 2.85 \\
\hline
\end{tabular}


Table A.2. Hyperfine structure of Sc and Co.

\begin{tabular}{|c|c|c|c|c|c|c|c|c|c|c|c|}
\hline Specie & $\lambda(\mathrm{nm})$ & $\chi(\mathrm{eV})$ & $\log g f$ & Specie & $\lambda(\mathrm{nm})$ & $\chi(\mathrm{eV})$ & $\log g f$ & Specie & $\lambda(\mathrm{nm})$ & $\chi(\mathrm{eV})$ & $\log g f$ \\
\hline Sc II & 361.3815 & 0.022 & -0.126 & CoI & 341.2328 & 0.514 & -0.171 & CoI & 350.2241 & 0.432 & -0.221 \\
\hline Sc II & 361.3814 & 0.022 & -0.141 & CoI & 341.2325 & 0.514 & -0.151 & CoI & 350.2263 & 0.432 & -0.110 \\
\hline Sc II & 361.3819 & 0.022 & -0.174 & CoI & 341.2333 & 0.514 & -0.241 & CoI & 350.2255 & 0.432 & -0.132 \\
\hline Sc II & 361.3817 & 0.022 & -0.108 & CoI & 341.2330 & 0.514 & -0.151 & CoI & 350.2245 & 0.432 & -0.231 \\
\hline Sc II & 361.3815 & 0.022 & -0.107 & CoI & 341.2325 & 0.514 & -0.131 & Co I & 350.2272 & 0.432 & -0.095 \\
\hline Sc II & 361.3823 & 0.022 & -0.163 & CoI & 341.2338 & 0.514 & -0.225 & CoI & 350.2262 & 0.432 & -0.133 \\
\hline Sc II & 361.3821 & 0.022 & -0.096 & CoI & 341.2333 & 0.514 & -0.141 & CoI & 350.2251 & 0.432 & -0.251 \\
\hline Sc II & 361.3817 & 0.022 & -0.083 & CoI & 341.2326 & 0.514 & -0.114 & CoI & 350.2283 & 0.432 & -0.082 \\
\hline Sc II & 361.3828 & 0.022 & -0.165 & CoI & 341.2343 & 0.514 & -0.225 & CoI & 350.2271 & 0.432 & -0.140 \\
\hline Sc II & 361.3825 & 0.022 & -0.090 & CoI & 341.2336 & 0.514 & -0.136 & CoI & 350.2257 & 0.432 & -0.291 \\
\hline Sc II & 361.3821 & 0.022 & -0.065 & CoI & 341.2328 & 0.514 & -0.099 & CoI & 350.2295 & 0.432 & -0.071 \\
\hline Sc II & 361.3835 & 0.022 & -0.176 & CoI & 341.2349 & 0.514 & -0.235 & CoI & 350.2281 & 0.432 & -0.161 \\
\hline Sc II & 361.3831 & 0.022 & -0.089 & CoI & 341.2341 & 0.514 & -0.137 & CoI & 350.2308 & 0.432 & -0.060 \\
\hline Sc II & 361.3826 & 0.022 & -0.049 & CoI & 341.2330 & 0.514 & -0.086 & & & & \\
\hline Sc II & 361.3843 & 0.022 & -0.198 & CoI & 341.2356 & 0.514 & -0.256 & CoI & 384.5473 & 0.923 & -0.180 \\
\hline Sc II & 361.3838 & 0.022 & -0.096 & CoI & 341.2346 & 0.514 & -0.144 & CoI & 384.5475 & 0.923 & -0.173 \\
\hline Sc II & 361.3832 & 0.022 & -0.036 & CoI & 341.2334 & 0.514 & -0.075 & CoI & 384.5470 & 0.923 & -0.153 \\
\hline Sc II & 361.3853 & 0.022 & -0.238 & CoI & 341.2364 & 0.514 & -0.295 & CoI & 384.5479 & 0.923 & -0.243 \\
\hline Sc II & 361.3847 & 0.022 & -0.117 & CoI & 341.2352 & 0.514 & -0.165 & CoI & 384.5474 & 0.923 & -0.153 \\
\hline \multirow[t]{2}{*}{ Sc II } & 361.3840 & 0.022 & -0.024 & CoI & 341.2338 & 0.514 & -0.064 & CoI & 384.5468 & 0.923 & -0.133 \\
\hline & & & & & & & & Co I & 384.5480 & 0.923 & -0.227 \\
\hline Sc II & 424.6832 & 0.315 & -0.096 & CoI & 344.9178 & 0.581 & -0.174 & CoI & 384.5474 & 0.923 & -0.143 \\
\hline Sc II & 424.6836 & 0.315 & -0.080 & CoI & 344.9172 & 0.581 & -0.149 & Co I & 384.5465 & 0.923 & -0.116 \\
\hline Sc II & 424.6836 & 0.315 & -0.039 & CoI & 344.9183 & 0.581 & -0.149 & CoI & 384.5481 & 0.923 & -0.227 \\
\hline Sc II & 424.6839 & 0.315 & -0.081 & CoI & 344.9176 & 0.581 & -0.339 & CoI & 384.5473 & 0.923 & -0.138 \\
\hline Sc II & 424.6839 & 0.315 & -0.078 & CoI & 344.9167 & 0.581 & -0.129 & CoI & 384.5462 & 0.923 & -0.101 \\
\hline Sc II & 424.6841 & 0.315 & -0.098 & CoI & 344.9183 & 0.581 & -0.129 & CoI & 384.5482 & 0.923 & -0.237 \\
\hline Sc II & 424.6842 & 0.315 & -0.148 & CoI & 344.9173 & 0.581 & -0.215 & CoI & 384.5471 & 0.923 & -0.139 \\
\hline Sc II & 424.6843 & 0.315 & -0.291 & CoI & 344.9161 & 0.581 & -0.122 & CoI & 384.5458 & 0.923 & -0.088 \\
\hline Sc II & 424.6843 & 0.315 & -0.096 & CoI & 344.9182 & 0.581 & -0.122 & CoI & 384.5483 & 0.923 & -0.257 \\
\hline Sc II & 424.6844 & 0.315 & -0.116 & CoI & 344.9170 & 0.581 & -0.143 & CoI & 384.5470 & 0.923 & -0.146 \\
\hline Sc II & 424.6845 & 0.315 & -0.080 & CoI & 344.9154 & 0.581 & -0.125 & CoI & 384.5455 & 0.923 & -0.077 \\
\hline Sc II & 424.6846 & 0.315 & -0.098 & CoI & 344.9181 & 0.581 & -0.125 & CoI & 384.5483 & 0.923 & -0.297 \\
\hline \multirow[t]{2}{*}{ Sc II } & 424.6846 & 0.315 & -0.081 & CoI & 344.9165 & 0.581 & -0.103 & CoI & 384.5468 & 0.923 & -0.167 \\
\hline & & & & CoI & 344.9146 & 0.581 & -0.144 & Co I & 384.5450 & 0.923 & -0.066 \\
\hline CoI & 340.5074 & 0.432 & -0.139 & CoI & 344.9178 & 0.581 & -0.144 & & & & \\
\hline CoI & 340.5072 & 0.432 & -0.159 & CoI & 344.9159 & 0.581 & -0.074 & CoI & 399.5299 & 0.923 & -0.203 \\
\hline CoI & 340.5081 & 0.432 & -0.159 & & & & & CoI & 399.5304 & 0.923 & -0.203 \\
\hline CoI & 340.5079 & 0.432 & -0.138 & CoI & 345.3472 & 0.432 & -0.105 & CoI & 399.5302 & 0.923 & -0.305 \\
\hline CoI & 340.5075 & 0.432 & -0.137 & CoI & 345.3479 & 0.432 & -0.150 & CoI & 399.5297 & 0.923 & -0.175 \\
\hline CoI & 340.5088 & 0.432 & -0.137 & CoI & 345.3475 & 0.432 & -0.093 & $\mathrm{CoI}$ & 399.5306 & 0.923 & -0.175 \\
\hline CoI & 340.5085 & 0.432 & -0.124 & CoI & 345.3489 & 0.432 & -0.250 & CoI & 399.5302 & 0.923 & -0.235 \\
\hline Co I & 340.5081 & 0.432 & -0.126 & CoI & 345.3485 & 0.432 & -0.129 & Co I & 399.5296 & 0.923 & -0.161 \\
\hline CoI & 340.5098 & 0.432 & -0.126 & CoI & 345.3480 & 0.432 & -0.081 & CoI & 399.5308 & 0.923 & -0.161 \\
\hline Co I & 340.5093 & 0.432 & -0.107 & CoI & 345.3498 & 0.432 & -0.229 & CoI & 399.5302 & 0.923 & -0.190 \\
\hline Co I & 340.5088 & 0.432 & -0.122 & CoI & 345.3493 & 0.432 & -0.119 & CoI & 399.5294 & 0.923 & -0.154 \\
\hline Co I & 340.5110 & 0.432 & -0.122 & CoI & 345.3488 & 0.432 & -0.070 & Co I & 399.5310 & 0.923 & -0.154 \\
\hline CoI & 340.5104 & 0.432 & -0.090 & CoI & 345.3510 & 0.432 & -0.226 & CoI & 399.5302 & 0.923 & -0.157 \\
\hline CoI & 340.5097 & 0.432 & -0.123 & CoI & 345.3504 & 0.432 & -0.115 & CoI & 399.5292 & 0.923 & -0.153 \\
\hline CoI & 340.5123 & 0.432 & -0.123 & CoI & 345.3497 & 0.432 & -0.059 & CoI & 399.5313 & 0.923 & -0.153 \\
\hline Co I & 340.5116 & 0.432 & -0.074 & CoI & 345.3524 & 0.432 & -0.234 & CoI & 399.5302 & 0.923 & -0.131 \\
\hline CoI & 340.5108 & 0.432 & -0.131 & CoI & 345.3517 & 0.432 & -0.117 & CoI & 399.5290 & 0.923 & -0.160 \\
\hline CoI & 340.5139 & 0.432 & -0.131 & CoI & 345.3508 & 0.432 & -0.050 & CoI & 399.5315 & 0.923 & -0.160 \\
\hline Co I & 340.5131 & 0.432 & -0.060 & CoI & 345.3540 & 0.432 & -0.253 & CoI & 399.5302 & 0.923 & -0.109 \\
\hline CoI & 340.5121 & 0.432 & -0.152 & CoI & 345.3531 & 0.432 & -0.125 & CoI & 399.5288 & 0.923 & -0.180 \\
\hline Co I & 340.5156 & 0.432 & -0.152 & CoI & 345.3522 & 0.432 & -0.041 & CoI & 399.5317 & 0.923 & -0.180 \\
\hline Co I & 340.5147 & 0.432 & -0.046 & CoI & 345.3558 & 0.432 & -0.292 & Co I & 399.5302 & 0.923 & -0.090 \\
\hline & & & & CoI & 345.3548 & 0.432 & -0.147 & & & & \\
\hline CoI & 340.9155 & 0.514 & -0.204 & CoI & 345.3537 & 0.432 & -0.032 & CoI & 412.1330 & 0.923 & -0.213 \\
\hline Co I & 340.9159 & 0.514 & -0.204 & & & & & Co I & 412.1332 & 0.923 & -0.206 \\
\hline CoI & 340.9157 & 0.514 & -0.306 & CoI & 349.5710 & 0.629 & -0.147 & CoI & 412.1326 & 0.923 & -0.186 \\
\hline Co I & 340.9154 & 0.514 & -0.176 & CoI & 349.5703 & 0.629 & -0.147 & CoI & 412.1337 & 0.923 & -0.276 \\
\hline CoI & 340.9162 & 0.514 & -0.176 & CoI & 349.5693 & 0.629 & -0.177 & CoI & 412.1331 & 0.923 & -0.186 \\
\hline Co I & 340.9159 & 0.514 & -0.236 & CoI & 349.5707 & 0.629 & -0.165 & CoI & 412.1323 & 0.923 & -0.166 \\
\hline CoI & 340.9155 & 0.514 & -0.162 & CoI & 349.5697 & 0.629 & -0.131 & CoI & 412.1338 & 0.923 & -0.260 \\
\hline Co I & 340.9167 & 0.514 & -0.162 & CoI & 349.5684 & 0.629 & -0.134 & CoI & 412.1329 & 0.923 & -0.176 \\
\hline Co I & 340.9163 & 0.514 & -0.191 & CoI & 349.5702 & 0.629 & -0.190 & Co I & 412.1318 & 0.923 & -0.149 \\
\hline CoI & 340.9157 & 0.514 & -0.155 & CoI & 349.5689 & 0.629 & -0.130 & CoI & 412.1338 & 0.923 & -0.260 \\
\hline Co I & 340.9173 & 0.514 & -0.155 & CoI & 349.5673 & 0.629 & -0.105 & CoI & 412.1327 & 0.923 & -0.171 \\
\hline Co I & 340.9167 & 0.514 & -0.158 & CoI & 349.5695 & 0.629 & -0.233 & CoI & 412.1313 & 0.923 & -0.134 \\
\hline Co I & 340.9160 & 0.514 & -0.154 & CoI & 349.5679 & 0.629 & -0.146 & CoI & 412.1338 & 0.923 & -0.270 \\
\hline CoI & 340.9180 & 0.514 & -0.154 & CoI & 349.5659 & 0.629 & -0.084 & CoI & 412.1324 & 0.923 & -0.172 \\
\hline CoI & 340.9173 & 0.514 & -0.132 & & & & & Co I & 412.1308 & 0.923 & -0.121 \\
\hline CoI & 340.9164 & 0.514 & -0.161 & CoI & 350.2243 & 0.432 & -0.174 & CoI & 412.1338 & 0.923 & -0.290 \\
\hline Co I & 340.9188 & 0.514 & -0.161 & CoI & 350.2242 & 0.432 & -0.167 & CoI & 412.1321 & 0.923 & -0.179 \\
\hline CoI & 340.9179 & 0.514 & -0.110 & CoI & 350.2238 & 0.432 & -0.237 & CoI & 412.1301 & 0.923 & -0.110 \\
\hline CoI & 340.9169 & 0.514 & -0.181 & CoI & 350.2248 & 0.432 & -0.147 & CoI & 412.1336 & 0.923 & -0.330 \\
\hline CoI & 340.9197 & 0.514 & -0.181 & CoI & 350.2245 & 0.432 & -0.147 & CoI & 412.1316 & 0.923 & -0.200 \\
\hline CoI & 340.9187 & 0.514 & -0.091 & CoI & 350.2239 & 0.432 & -0.221 & CoI & 412.1294 & 0.923 & -0.099 \\
\hline & & & & CoI & 350.2255 & 0.432 & -0.127 & & & & \\
\hline CoI & 341.2325 & 0.514 & -0.178 & CoI & 350.2249 & 0.432 & -0.137 & & & & \\
\hline
\end{tabular}

